\title{
The mapping of the local contributions of Fermi and Coulomb correlation into intracule and extracule density distributions
}

\author{
Xavier Fradera and Miquel Duran a) \\ Institute of Computational Chemistry, University of Girona, 17071 Girona, Catalonia \\ Jordi Mestres ${ }^{\text {b) }}$ \\ Molecular Design \& Informatics, N.V. Organon, Molenstraat 110, P.O. Box 20, \\ 5340 BH Oss, The Netherlands
}

(Received 8 March 2000; accepted 18 May 2000)

\begin{abstract}
The contributions of the correlated and uncorrelated components of the electron-pair density to atomic and molecular intracule $I(\mathbf{r})$ and extracule $E(\mathbf{R})$ densities and its Laplacian functions $\nabla^{2} I(\mathbf{r})$ and $\nabla^{2} E(\mathbf{R})$ are analyzed at the Hartree-Fock (HF) and configuration interaction (CI) levels of theory. The topologies of the uncorrelated components of these functions can be rationalized in terms of the corresponding one-electron densities. In contrast, by analyzing the correlated components of $I(\mathbf{r})$ and $E(\mathbf{R})$, namely, $I^{C}(\mathbf{r})$ and $E^{C}(\mathbf{R})$, the effect of electron Fermi and Coulomb correlation can be assessed at the HF and CI levels of theory. Moreover, the contribution of Coulomb correlation can be isolated by means of difference maps between $I^{C}(\mathbf{r})$ and $E^{C}(\mathbf{R})$ distributions calculated at the two levels of theory. As application examples, the $\mathrm{He}, \mathrm{Ne}$, and $\mathrm{Ar}$ atomic series, the $\mathrm{C}_{2}^{-2}, \mathrm{~N}_{2}, \mathrm{O}_{2}^{+2}$ molecular series, and the $\mathrm{C}_{2} \mathrm{H}_{4}$ molecule have been investigated. For these atoms and molecules, it is found that Fermi correlation accounts for the main characteristics of $I^{C}(\mathbf{r})$ and $E^{C}(\mathbf{R})$, with Coulomb correlation increasing slightly the locality of these functions at the CI level of theory. Furthermore, $I^{C}(\mathbf{r}), E^{C}(\mathbf{R})$, and the associated Laplacian functions, reveal the short-ranged nature and high isotropy of Fermi and Coulomb correlation in atoms and molecules. (C) 2000 American Institute of Physics. [S0021-9606(00)30631-6]
\end{abstract}

\section{INTRODUCTION}

In the last years, the topological analysis of the oneelectron density $\rho(\mathbf{r})$ has become a widely used tool for analyzing the electronic structure of atoms and molecules. ${ }^{1}$ Recently, the analysis of the electron-pair density and related functions has also received increasing attention. ${ }^{2-21}$ However, the fact that the electron-pair density $\Gamma\left(\mathbf{r}_{1}, \mathbf{r}_{2}\right)$ is a six-dimensional function, ${ }^{2}$ makes it difficult to visualize and analyze directly. The analysis of electron intracule and extracule densities instead of the electron-pair density itself appears to be an interesting alternative. Intracule and extracule densities have the advantage of having a lower dimensionality than $\Gamma\left(\mathbf{r}_{1}, \mathbf{r}_{2}\right)$ while, at the same time, they keep a twoelectron character. ${ }^{3}$

For any pair of electrons, one can define the intracule and extracule coordinates as $\mathbf{r}=\mathbf{r}_{1}-\mathbf{r}_{2}$, and $\mathbf{R}=\left(\mathbf{r}_{1}+\mathbf{r}_{2}\right) / 2$, respectively. Then, intracule and extracule densities can be expressed $\mathrm{as}^{3}$

$$
I(\mathbf{r})=\int \Gamma\left(\mathbf{r}_{1}, \mathbf{r}_{2}\right) \delta\left(\left(\mathbf{r}_{1}-\mathbf{r}_{2}\right)-r\right) d \mathbf{r}_{1} d \mathbf{r}_{2},
$$

and

$$
E(\mathbf{R})=\int \Gamma\left(\mathbf{r}_{1}, \mathbf{r}_{2}\right) \delta\left(\frac{\mathbf{r}_{1}+\mathbf{r}_{2}}{2}-\mathbf{R}\right) d \mathbf{r}_{1} d \mathbf{r}_{2},
$$

\footnotetext{
${ }^{\text {a) Electronic mail: quel@stark.udg.es }}$

b) Author to whom correspondence should be addressed. Electronic mail: j.mestres@organon.oss.akzonobel.nl
}

respectively. $I(\mathbf{r})$ and $E(\mathbf{R})$ are probability density functions for the interelectronic separation vector and for the center of mass of the electron pair, respectively. Both $I(\mathbf{r})$ and $E(\mathbf{R})$ integrate to the number of electron pairs in the system. Besides, $I(\mathbf{r})$ has the property of being invariant to translations of the molecule, and always has a center of inversion at the origin. On the other hand, $E(\mathbf{R})$ has the same symmetry as $\rho(\mathbf{r})$, and its origin is also dependent on molecular translations. An additional property of $I(\mathbf{r})$ is that it must obey an electron-electron cusp condition at the origin. ${ }^{4}$ However, this cusp condition is not satisfied by approximate $I(\mathbf{r})$ densities calculated at the Hartree-Fock (HF) level of theory. In fact, in order to obtain $I(\mathbf{r})$ densities satisfying the electronelectron cusp condition, one has to use rather accurate wave functions, generated with explicit consideration of interelectronic distances. ${ }^{5}$

Until recently, topological analyses of $I(\mathbf{r})$ and $E(\mathbf{R})$ densities were restricted mainly to atoms and diatomic molecules (see Ref. 6 for a recent review). Early studies pointed that the interpretation of intracule and extracule densities should be based on the identification of the different kinds of electron-electron interactions possible in atoms and molecules. ${ }^{7-9}$ Moreover, the usefulness of the contracted electron-pair densities for the study of electron correlation was also made evident soon. In particular, the radial intracule density was used to study Fermi and Coulomb correlation by means of the corresponding density holes. ${ }^{10,11}$

In the last years, several topological analyses of $I(\mathbf{r})$ and $E(\mathbf{R})$ densities and their Laplacian functions $\nabla^{2} I(\mathbf{r})$ and 
$\nabla^{2} E(\mathbf{R})$ have been performed for polyatomic molecules. ${ }^{12-15}$ In general, these functions show topologies far more complex than those of the corresponding oneelectron densities or Laplacians. However, for $I(\mathbf{r})$ and $E(\mathbf{R})$ distributions calculated at the HF and configuration interaction (CI) levels of theory, the main topological features can be easily interpreted by using a simple scheme involving formal electron-electron interactions. ${ }^{13,14}$ Thus, local maxima in $I(\mathbf{r})$ and $E(\mathbf{R})$, or local minima in $\nabla^{2} I(\mathbf{r})$ and $\nabla^{2} E(\mathbf{R})$, can be associated to interactions between electrons formally assigned to the same or to different atoms. Furthermore, $\nabla^{2} I(\mathbf{r})$ and $\nabla^{2} E(\mathbf{R})$ allow us to distinguish the contributions of core and valence electrons. ${ }^{13}$

The analysis of $I(\mathbf{r})$ and $E(\mathbf{R})$ and the corresponding Laplacian functions appear as a promising tool for investigating molecular electronic structure from an electron-pair perspective. However, while most of the topological features appearing in the one-electron density $\rho(\mathbf{r})$ can be given a physical significance, this is not so for $I(\mathbf{r})$ and $E(\mathbf{R})$. For instance, maxima and saddle points in $\rho(\mathbf{r})$ correspond to nuclear attractors and bond critical points, respectively. These topological features, together with the gradient of the density $\nabla \rho(\mathbf{r})$, can be used to define atoms in real space, and to decide whether any two atoms have a chemical bond between them. ${ }^{1}$ On the other hand, the existence of a local maximum in $I(\mathbf{r})$ or $E(\mathbf{R})$ assigned to an electron-electron interaction, does not mean that the electrons involved have a strong physical interaction between them. Also, the interpretation of saddle points and other topological features in $I(\mathbf{r})$ or $E(\mathbf{R})$ distributions is not clear at the moment, except for the region around the origin in $I(\mathbf{r}) \cdot{ }^{15}$ In addition, depending on the size and symmetry of the molecule, different electron-electron interactions can contribute to the same point in space, making it impossible to assess the individual contribution of each interaction to $I(\mathbf{r})$ and $E(\mathbf{R}){ }^{13,14}$

In order to gain insight into the nature of electronelectron interactions in atoms and molecules, and to analyze the effects of Fermi and Coulomb electron correlation on contracted electron-pair densities, we propose to study separately the correlated and uncorrelated components of $I(\mathbf{r})$ and $E(\mathbf{R})$

$$
\Gamma\left(\mathbf{r}_{1}, \mathbf{r}_{2}\right)=\Gamma^{U}\left(\mathbf{r}_{1}, \mathbf{r}_{2}\right)+\Gamma^{C}\left(\mathbf{r}_{1}, \mathbf{r}_{2}\right) .
$$

The uncorrelated component of the electron-pair density $\Gamma^{U}\left(\mathbf{r}_{1}, \mathbf{r}_{2}\right)$ is a product of two one-electron densities

$$
\Gamma^{U}\left(\mathbf{r}_{1}, \mathbf{r}_{2}\right)=\frac{\rho\left(\mathbf{r}_{1}\right) \rho\left(\mathbf{r}_{2}\right)}{2},
$$

and integrates to $N^{2} / 2$, where $N$ is the number of electrons in the atom or molecule. In turn, $\Gamma^{C}\left(\mathbf{r}_{1}, \mathbf{r}_{2}\right)$ is the correlated component of the electron-pair density

$$
\Gamma^{C}\left(\mathbf{r}_{1}, \mathbf{r}_{2}\right)=\Gamma\left(\mathbf{r}_{1}, \mathbf{r}_{2}\right)-\frac{\rho\left(\mathbf{r}_{1}\right) \rho\left(\mathbf{r}_{2}\right)}{2}=\frac{f\left(\mathbf{r}_{1}, \mathbf{r}_{2}\right)}{2},
$$

and integrates to $-N / 2$. Thus, $\Gamma^{C}\left(\mathbf{r}_{1}, \mathbf{r}_{2}\right)$ compensates for the electron self-interactions present in $\Gamma^{U}\left(\mathbf{r}_{1}, \mathbf{r}_{2}\right)$ in order for the electron-pair density to integrate to the correct number of $\left(N^{2}-N\right) / 2$ electron pairs. Besides, $\Gamma^{C}\left(\mathbf{r}_{1}, \mathbf{r}_{2}\right)$ accounts also for Fermi and Coulomb correlation between electrons. At the HF level of theory, only Fermi correlation, or exchange, between same-spin electrons is considered. In this case, one can call the function $f\left(\mathbf{r}_{1}, \mathbf{r}_{2}\right)$ used in Eq. (5) the exchange or Fermi density. On the contrary, at higher levels of theory, Coulomb correlation is also taken into account. Then, in general, one can call $f\left(\mathbf{r}_{1}, \mathbf{r}_{2}\right)$ the exchangecorrelation density. $f\left(\mathbf{r}_{1}, \mathbf{r}_{2}\right)$ is the basis for the definition of Fermi or exchange-correlation hole densities, ${ }^{17}$ which can be used to visualize the degree of electron localization at arbitrary positions of real space. ${ }^{18}$ Within the framework of the theory of atoms in molecules, ${ }^{1} f\left(\mathbf{r}_{1}, \mathbf{r}_{2}\right)$ determines the degree of electron localization in atoms and electron delocalization between pairs of atoms. ${ }^{19,20}$

The uncorrelated and correlated components of the electron-pair density are also six-dimensional functions. Therefore, Eqs. (1) and (2) can be applied to $\Gamma^{U}\left(\mathbf{r}_{1}, \mathbf{r}_{2}\right)$ and $\Gamma^{C}\left(\mathbf{r}_{1}, \mathbf{r}_{2}\right)$, in order to obtain the uncorrelated, $I^{U}(\mathbf{r})$, $E^{U}(\mathbf{R})$, and correlated $I^{C}(\mathbf{r}), E^{C}(\mathbf{R})$ components of the Intracule and extracule densities, as well as the associated Laplacian functions. Thus, the correlated and uncorrelated components of the radial intracule density have been calculated recently for a number of molecules at the HF level of theory. ${ }^{21}$ Note that, within the HF approximation, the Coulomb and exchange components defined in Ref. 21 correspond to the uncorrelated and correlated components, respectively, according to the general terminology used throughout the present paper. The analysis of the uncorrelated component of the radial intracule density revealed the long-range nature of electrostatic interactions, while the correlated component showed the short-range nature of exchange effects in the electron-pair density. Moreover, it was found that delocalization effects are also reflected on the correlated radial intracule density.

This paper deals with the correlated and uncorrelated components of the intracule and extracule distributions of several atoms and molecules. Our aim is to investigate for the first time the topology of these functions, focusing the analysis on the changes caused by introduction of Fermi and Coulomb correlation on the uncorrelated intracule and extracule densities. As application examples, the $\mathrm{He}, \mathrm{Ne}$, and $\mathrm{Ar}$ atoms, the $\mathrm{C}_{2}^{-2}, \mathrm{~N}_{2}$, and $\mathrm{O}_{2}^{+2}$ series of molecules, and the $\mathrm{C}_{2} \mathrm{H}_{4}$ molecule have been selected. In order to analyze separately the effects of Fermi and Coulomb correlation on the contracted pair densities, $\mathrm{HF}$ and $\mathrm{CI}$ results are presented for the three series. For $\mathrm{C}_{2} \mathrm{H}_{4}$, the results presented in this paper complement the analysis done previously at the HF level of theory for the same molecule. ${ }^{13}$

\section{COMPUTATIONAL METHODS}

Calculations for the $\mathrm{He}, \mathrm{Ne}$, and $\mathrm{Ar}$ atoms were carried out at the HF/6-311G* and CISD/6-311G* levels of theory, placing each atom at the origin of coordinates. The molecular geometries of $\mathrm{C}_{2}^{-2}, \mathrm{~N}_{2}$, and $\mathrm{O}_{2}^{+2}$ were optimized at the $\mathrm{HF} / 6-311+\mathrm{G}(2 d)$ level of theory, whereas for $\mathrm{C}_{2} \mathrm{H}_{4}$, $\mathrm{HF} / 6-31 \mathrm{G}^{*}$ optimized geometrical parameters were taken 
from Ref. 13. Then, CISD/6-311+ G(2d) (for $\mathrm{C}_{2}^{-2}, \mathrm{~N}_{2}$, and $\mathrm{O}_{2}^{+2}$ ) and CISD/6-31G* (for $\mathrm{C}_{2} \mathrm{H}_{4}$ ) wave functions were computed, using the HF-optimized geometries. All molecular coordinates were mass centered, and the heavy atoms of each molecule were placed along the $x$ axis. All calculations were performed with the GAMESS package. ${ }^{22}$ For the HF calculations, second-order density matrix elements were generated straightforwardly from the first-order ones, while for CI calculations, second-order density matrix elements were generated by GAMESS.

For all levels of theory, density matrix elements corresponding to the uncorrelated and correlated parts of the electron-pair density were obtained according to the following equations:

$$
\begin{aligned}
& D_{\mu \nu \lambda \sigma}^{U}=\frac{D_{\mu \nu} D_{\lambda \sigma}}{2}, \\
& D_{\mu \nu \lambda \sigma}^{C}=D_{\mu \nu \lambda \sigma}-\frac{D_{\mu \nu} D_{\lambda \sigma}}{2},
\end{aligned}
$$

where $D_{\mu \nu}$ and $D_{\mu \nu \lambda \sigma}$ are the first- and second-order density matrix elements, respectively, and $D_{\mu \nu \lambda \sigma}^{U}$ and $D_{\mu \nu \lambda \sigma}^{C}$ are the uncorrelated and correlated components of the second order density matrix, respectively.

For each of the two-electron densities generated, calculations of intracule and extracule distributions and their respective Laplacians were performed following the algorithm described in Ref. 23, using an integral neglect threshold of $10^{-5}$. For the molecules, electron-electron interactions were characterized by locating local minima in the Laplacian functions [maxima in $\nabla^{2} I^{C}(\mathbf{r})$ and $\left.\nabla^{2} E^{C}(\mathbf{R})\right]$. In order to associate an electron-electron interaction to a local maximum or minimum, we considered a maximum distance threshold of 0.5 a.u. (for intracule distributions) or 0.25 a.u. (for extracule distributions) between the actual position of the minimum or maximum and the position expected for that interaction according to the nuclear geometry. Maxima or minima not related to any formal electron-electron interaction, according to this criterium, are not reported. ${ }^{24}$

\section{RESULTS AND DISCUSSION}

This section reports the results for the $\mathrm{He}, \mathrm{Ne}$, and $\mathrm{Ar}$ atoms, the $\mathrm{C}_{2}^{-2}, \mathrm{~N}_{2}$, and $\mathrm{O}_{2}^{+2}$ molecules, and $\mathrm{C}_{2} \mathrm{H}_{4}$, which have been selected as application examples. In general, for the three series, the analysis will focus on the topological characteristics of the contracted electron-pair densities and its correlated and uncorrelated components. Moreover, comparative analysis between the results obtained at the HF and CI levels of theory will also be performed.

In order to aid in the interpretation of the results, Scheme 1 depicts the relationship between the total electronpair density $\Gamma\left(\mathbf{r}_{1}, \mathbf{r}_{2}\right)$ or $\Gamma$ for short, and its components. The different components of $\Gamma$ (uncorrelated and correlated) as well as the total pair density are represented along the vertical axis, while the level of calculation is represented along

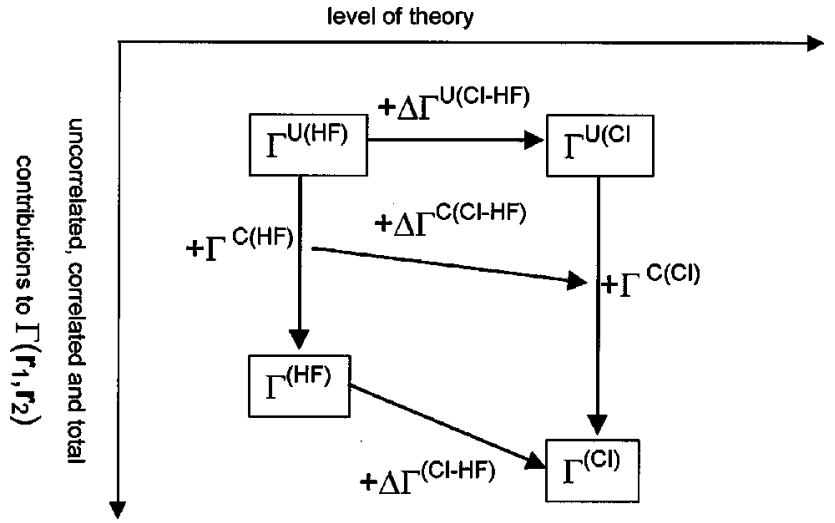

Scheme 1.

the horizontal axis. For any level of theory, $\Gamma^{U}$ is the uncorrelated component of $\Gamma$, with no direct inclusion of any kind of electron correlation. Addition of the correlated component $\Gamma^{C}$ yields $\Gamma$, the total electron-pair density. $\Delta \Gamma^{U(\mathrm{CI}-\mathrm{HF})}$, $\Delta \Gamma^{C(\mathrm{CI}-\mathrm{HF})}$, and $\Delta \Gamma^{(\mathrm{CI}-\mathrm{HF})}$ stand for the $\Gamma^{U}, \Gamma^{C}$, and $\Gamma$ CI-HF differences, respectively. Note that in order to allow for a straightforward comparison of $\mathrm{HF}$ and CISD contracted-electron pair densities, all molecular CI calculations have been performed at the HF optimized geometries (see above). The vertical positions of the HF and CI densities in the scheme reflect that $\Gamma^{C(\mathrm{HF})}$ accounts only for Fermi correlation, while $\Gamma^{C(\mathrm{CI})}$ includes Fermi and Coulomb correlation. Accordingly, considering that Fermi correlation is similar at the HF and CISD levels of theory, $\Delta \Gamma^{C(\mathrm{Cl}-\mathrm{HF})}$ can be considered to account mainly for the Coulomb correlation introduced at the CISD level. The same definitions stated for $\Gamma$ are valid also for each contracted electron-pair density, namely $I(\mathbf{r})$ and $E(\mathbf{R})$, as well as for the associated Laplacian functions.

There are a total number of nine topologies to analyze, including density functions and differences between density functions, for each choice of a system and electron-pair density (intracule or extracule). However, for the sake of brevity, only the most meaningful functions carrying information of interest are depicted and analyzed. Moreover, the compact notation introduced in Scheme 1 will be used from now on, using $I$ and $E$ instead of $\Gamma$ for intracule and extracule distributions, respectively. Accordingly, $\nabla^{2} I$ and $\nabla^{2} E$ will be used to refer to the corresponding Laplacian functions.

\section{A. Atomic series: $\mathrm{He}, \mathrm{Ne}, \mathrm{Ar}$}

Due to the spherical symmetry of these atoms, it is enough to study $I(\mathbf{r}), E(\mathbf{R})$, and its components along a radius starting at the nucleus of each atom. The first part of this analysis involves electron-pair densities obtained from HF wave functions. Thus, Figs. 1(a) and 1(b) contain radial plots of $I^{(\mathrm{HF})}$ and $E^{(\mathrm{HF})}$ for the Ne atom, as well as plots of the corresponding correlated and uncorrelated components. For the sake of comparison, $-I^{C(\mathrm{HF})}$ and $-E^{C(\mathrm{HF})}$ are plotted 


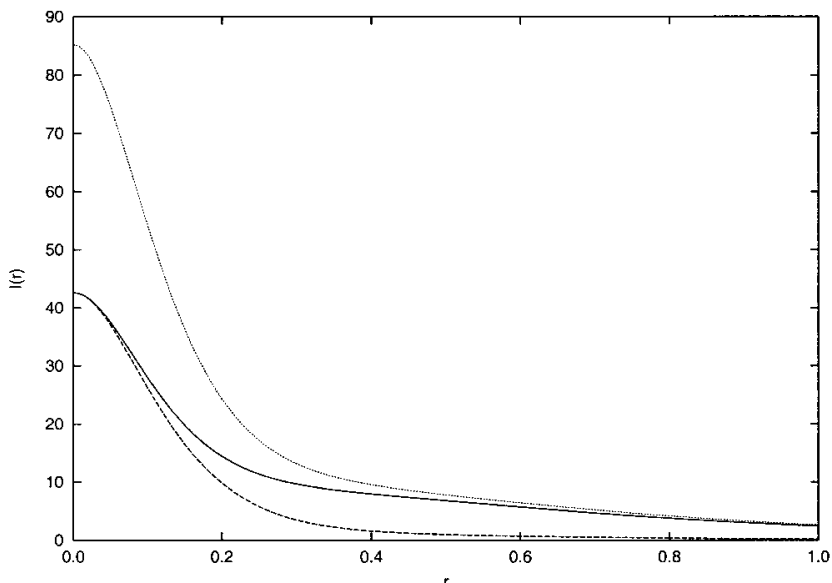

(a)

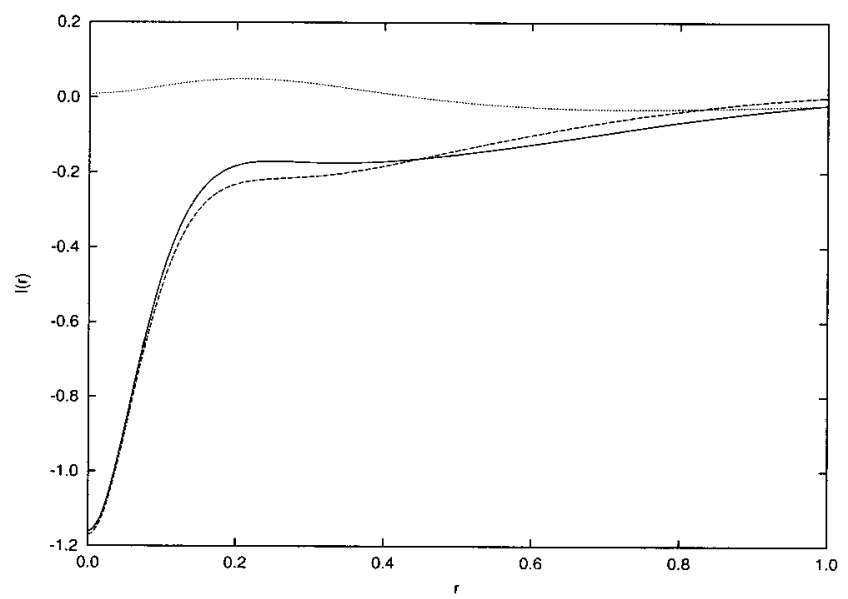

(c)

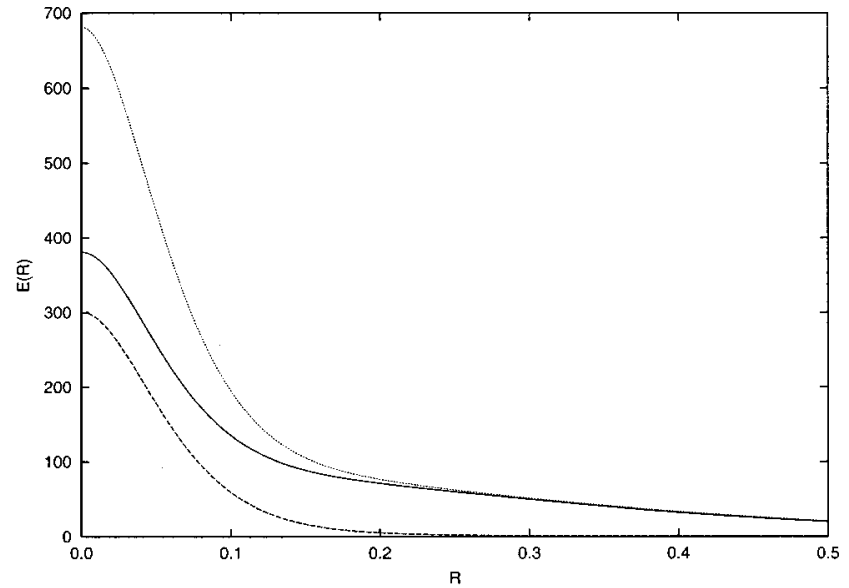

(b)

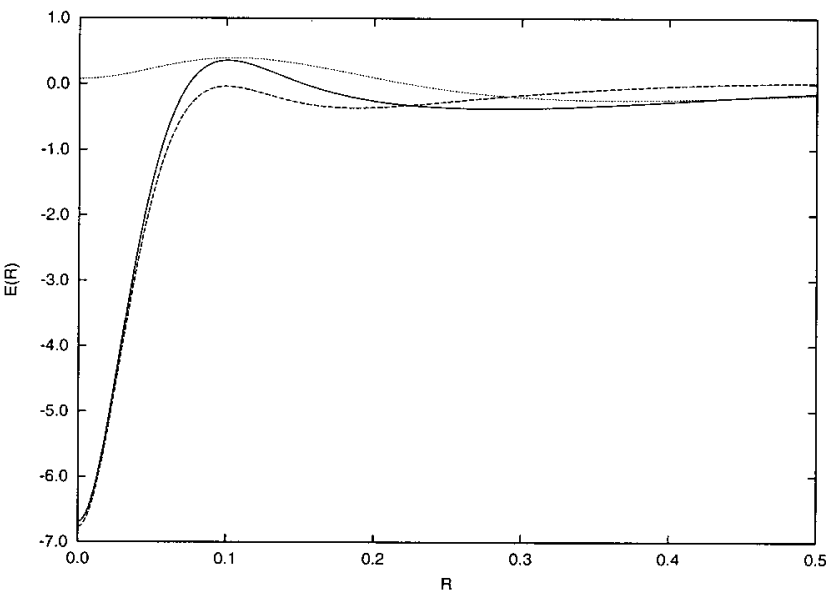

(d)

FIG. 1. HF $I(\mathbf{r})$ and $E(\mathbf{R})$ densities for Ne, starting at the nucleus, and differences between CI and HF $I(\mathbf{r})$ and $E(\mathbf{R})$ densities. Total densities are depicted in solid line, uncorrelated components in dotted line, and correlated components (with the sign changed) in dashed line: (a) $I^{(\mathrm{HF})}, I^{U(\mathrm{HF})}$, and $-I^{C(\mathrm{HF})}$ for Ne; (b) $E^{(\mathrm{HF})}, E^{U(\mathrm{HF})}$, and $-E^{C(\mathrm{HF})}$ for $\mathrm{Ne}$; (c) $\Delta I^{(\mathrm{CI}-\mathrm{HF})}, \Delta I^{U(\mathrm{CI}-\mathrm{HF})}$, and $-\Delta I^{C(\mathrm{Cl}-\mathrm{HF})}$ for $\mathrm{Ne}$; and (d) $\Delta E^{(\mathrm{CI}-\mathrm{HF})}, \Delta E^{U(\mathrm{Cl}-\mathrm{HF})}$, and $-\Delta E^{C(\mathrm{CI}-\mathrm{HF})}$ for $\mathrm{Ne}$.

instead of $I^{C(\mathrm{HF})}$ and $E^{C(\mathrm{HF})}$. Radial plots are presented for $\mathrm{Ne}$ only, because similar trends are found for $\mathrm{He}$ and Ar. When necessary, comparisons between the three atoms will be done by means of the values of $I(\mathbf{r}), E(\mathbf{R})$, and its components at the origin (see Table 1). $I(0)$ and $E(0)$ are the electron-electron coalescence and counterbalance densities, respectively. $^{25}$

$\mathrm{HF} / 6-311 \mathrm{G}^{*} I(0)$ and $E(0)$ values reported in Table I are relatively good approximations to the HF limit values calculated numerically, ${ }^{26}$ especially for $\mathrm{He}$ and $\mathrm{Ne}$. In con- trast, the CISD/6-311G* $I(0)$ and $E(0)$ values for $\mathrm{He}$ and $\mathrm{Ne}$ are not so close to those obtained by using highly accurate correlated wave functions. ${ }^{27-30}$ However, the general trends found between more accurate HF and correlated $I(0)$ and $E(0)$ values are preserved in Table I. Thus, both the coalescence and counterbalance densities decrease when considering Coulomb correlation at the CI level with respect to $\mathrm{HF}^{31}$

For $\mathrm{Ne}$, both $I^{(\mathrm{HF})}$ and $E^{(\mathrm{HF})}$ and its correlated and uncorrelated components are unimodal functions, with a single

TABLE I. $I(0)$ and $E(0)$ values for the $\mathrm{He}, \mathrm{Ne}$, and Ar atoms. Values (in a.u.) for the total densities $I(0)$ and $E(0)$, uncorrelated components $I^{U}(0)$ and $E^{U}(0)$, and correlated components $I^{C}(0)$ and $E^{C}(0)$ are presented. $\mathrm{HF}$ results are in roman type, and $\mathrm{CI}$ results in italics.

\begin{tabular}{lrrrrrr}
\hline \hline & \multicolumn{1}{c}{$I(0)$} & \multicolumn{1}{c}{$I^{U}(0)$} & \multicolumn{1}{c}{$I^{C}(0)$} & \multicolumn{1}{c}{$E(0)$} & \multicolumn{1}{c}{$E^{U}(0)$} & \multicolumn{1}{c}{$E^{C}(0)$} \\
\hline $\mathrm{He}$ & 0.190 & 0.381 & -0.190 & 1.524 & 3.047 & -1.524 \\
& 0.158 & 0.381 & -0.223 & 1.263 & 3.046 & -1.783 \\
$\mathrm{Ne}$ & 42.590 & 85.179 & -42.590 & 381.059 & 681.435 & -300.377 \\
& 41.430 & 85.189 & -43.759 & 374.367 & 681.511 & -307.144 \\
$\mathrm{Ar}$ & 302.344 & 604.689 & -302.344 & 2948.165 & 4837.511 & -1889.346 \\
& 299.088 & 604.544 & -305.456 & 2922.634 & 4836.351 & -1913.718 \\
\hline \hline
\end{tabular}


TABLE II. Total (correlated and uncorrelated) number of electron pairs contributing to the intracule and extracule shells of the Ne and Ar atoms. Intracule and extracule atomic shells are delimited by the even zeros in $\nabla^{2} I(\mathbf{r})$ and $\nabla^{2} E(\mathbf{R})$, respectively. $n_{1}, n_{2}$, and $n_{3}$ refer to the numbers of electron pairs in the first, second, and third shells. Values in roman type refer to HF/6-311+ $\mathrm{G}^{*}$ results; values in italics refer to CISD/6-311+ $\mathrm{G}^{*}$ results.

\begin{tabular}{|c|c|c|c|c|c|c|c|c|c|c|c|c|c|c|c|c|c|c|}
\hline \multirow[b]{3}{*}{ Atom } & \multicolumn{9}{|c|}{ Intracule shells } & \multicolumn{9}{|c|}{ Extracule shells } \\
\hline & \multicolumn{3}{|c|}{ Total } & \multicolumn{3}{|c|}{ Uncorrelated } & \multicolumn{3}{|c|}{ Correlated } & \multicolumn{3}{|c|}{ Total } & \multicolumn{3}{|c|}{ Uncorrelated } & \multicolumn{3}{|c|}{ Correlated } \\
\hline & $n_{1}$ & $n_{2}$ & $n_{3}$ & $n_{1}$ & $n_{2}$ & $n_{3}$ & $n_{1}$ & $n_{2}$ & $n_{3}$ & $n_{1}$ & $n_{2}$ & $n_{3}$ & $n_{1}$ & $n_{2}$ & $n_{3}$ & $n_{1}$ & $n_{2}$ & $n_{3}$ \\
\hline \multirow[t]{2}{*}{$\mathrm{Ne}$} & 2.404 & 42.599 & & 3.677 & 46.326 & & -1.273 & -3.727 & & 2.929 & 42.073 & & 3.855 & 46.148 & & -0.926 & -4.075 & \\
\hline & 2.420 & 42.582 & & 3.755 & 46.247 & & -1.335 & -3.665 & & 2.961 & 42.041 & & 3.899 & 46.104 & & -0.938 & -4.062 & \\
\hline \multirow[t]{2}{*}{$\mathrm{Ar}$} & 1.940 & 61.608 & 89.468 & 3.086 & 66.631 & 92.300 & -1.146 & -5.023 & -2.832 & 2.496 & 59.913 & 90.607 & 3.260 & 64.033 & 94.724 & -0.763 & -4.120 & -4.117 \\
\hline & 1.935 & 61.669 & 89.411 & 3.084 & 66.896 & 92.036 & -1.149 & -5.227 & -2.625 & 2.492 & 60.104 & 90.419 & 3.257 & 64.161 & 94.598 & -0.765 & -4.057 & -4.179 \\
\hline
\end{tabular}

maximum located at the origin of coordinates. The intracule graph [Fig. 1(a)] shows that Fermi correlation is maximal at the electron-electron coalescence point, and decays quickly with the interelectronic distance. Thus, for interelectronic distances larger than 0.6 a.u., $I^{(\mathrm{HF})}$ and $I^{U(\mathrm{HF})}$ are nearly equivalent. Another interesting feature is that, at the HF level, the following relationship holds for the coalescence density or intracule density at the origin: $I(0)=-I^{C}(0)$ $=I^{U}(0) / 2$. The reason is that for any atom or molecule, there are a total of $N^{2} / 2$ uncorrelated electron pairs contributing to some extent to $I^{U}(0)$ [see Eq. (4)]. Half these electron pairs have parallel spin, and, according to the antisymmetry principle, have a null contribution to the total $I(0)$ value. The other half are antiparallel electron pairs, which are totally uncorrelated at the HF level of theory, and make the same contribution to $I^{U}(0)$ and to $I(0)$. Therefore, the value of $I(0)$ is equal to $I^{U}(0) / 2$ and to $-I^{C}(0)$. This relationship also holds for $\mathrm{He}$ and $\mathrm{Ar}$ (Table I) and, in general, for closedshell systems within the HF approximation. As for the extracule graph [Fig. 1(b)], exchange correlation is strong only for pairs of electrons centered in the region around the origin, and decays quickly with the extracule coordinate. Thus, for values of $|\mathbf{R}|$ larger than 0.25 a.u., the contribution of $E^{C(\mathrm{HF})}$ to $E^{(\mathrm{HF})}$ is very small. However, in contrast to the equivalence of $I(0)$ and $-I^{C}(0)$ for the three atoms, $E(0)$ and $-E^{C}(0)$ are equivalent only for $\mathrm{He}$, while for $\mathrm{Ne}$ and $\mathrm{Ar}$ $E(0)$ is considerably larger than $-E^{C}(0)$ (Table I).

The intracule and extracule density distributions of $\mathrm{He}$ and Ar, not presented, follow the same trends found for Ne. However, for the $\mathrm{He}$ atom, $I^{(\mathrm{HF})}$ and $E^{(\mathrm{HF})}$ are exactly equivalent to $-I^{C(\mathrm{HF})}$ and $-E^{C(\mathrm{HF})}$, respectively, and also to $I^{U(\mathrm{HF})} / 2$ and $E^{U(\mathrm{HF})} / 2$, respectively, for all values of $\mathbf{r}$ or $\mathbf{R}$. The reason for this is that, at the HF level, the two antiparallel electrons in $\mathrm{He}$ are totally uncorrelated. Thus, the only effect of $\Gamma^{C}$, which integrates to -1 , is to correct for the self-interactions present in $\Gamma^{U}$, which integrates to 2 .

The second part of the analysis involves intracule and extracule densities obtained from post-HF wave functions. The effect of Coulomb correlation on the contracted electron-pair densities is apparent on the plots of differences between $\mathrm{CI}$ and $\mathrm{HF} I(\mathbf{r})$ and $E(\mathbf{R})$ densities, for the Ne atom [Figs. 1(c) and 1(d), respectively] and on the values at the origin for the three atoms (Table I). $\Delta I^{(\mathrm{CI}-\mathrm{HF})}$ and $\Delta E^{(\mathrm{CI}-\mathrm{HF})}$ present common trends for $\mathrm{He}, \mathrm{Ne}$, and Ar. First of all, the differences between $I^{U}$ distributions calculated at the HF and CI levels of theory are slight, compared to the differences obtained for $I$ or $I^{C}$. For instance, for the Ar atom, the difference between the HF and CI $I^{U}(0)$ values is only 0.15 a.u., while for $I^{C}(0)$ the difference is 3.1 a.u. Similar trends are also found for the extracule densities. These results show that, with respect to the HF reference, the electron-pair redistribution due to consideration of Coulomb correlation at the CI level is reflected mostly on the correlated component of the electron-pair density. The effect of increasing the level of calculation on one-electron densities is smaller; therefore the uncorrelated components of the contracted electron-pair densities also change little when considering Coulomb correlation.

CI-HF correlation shifts for $I$ and $E$ are very similar to those for $-I^{C}$ and $-E^{C}$. In all cases, upon consideration of Coulomb correlation, both intracule and extracule densities are shifted towards larger $\mathbf{r}$ or $\mathbf{R}$ coordinates, respectively. For the three atoms, maximal differences between the HF and CI results are found at the intracule and extracule origins of coordinates, and the difference decays quickly with increasing $\mathbf{r}$ or $\mathbf{R}$. At the coalescence point, Coulomb correlation increases the contribution of the correlated component with respect to the HF reference, and it is found that $I(0)$ $<I^{U}(0) / 2<-I^{C}(0)$ for all the atoms.

It is well known that the Laplacian of the electron density $\nabla^{2} \rho(\mathbf{r})$ is able to show the shell structure of atoms and molecules. ${ }^{1,32,33}$ Moreover, atomic shell structure has also been discussed from the point of view of the Laplacians of the intracule and extracule densities. ${ }^{34,35}$ Even though similar shell structures were found for $\nabla^{2} \rho(\mathbf{r}), \nabla^{2} I(\mathbf{r})$, and $\nabla^{2} E(\mathbf{R})$, the interpretation is different in each case. ${ }^{35}$ Table II collects the HF and CI electron-pair shell populations for the $\mathrm{Ne}$ and $\mathrm{Ar}$ atoms. At both levels of theory, $\mathrm{Ne}$ and $\mathrm{Ar}$ present two and three intracule shells, respectively. The same numbers of shells are found for the extracule densities of $\mathrm{Ne}$ and Ar. The uncorrelated and correlated electron-pair contributions to each intracule and extracule shell are also reported in Table II. These values show the importance of electron correlation for each shell.

$\mathrm{Ne}$ intracule populations are 2.40 and 42.60 at the HF level of theory, while extracule populations are 2.93 and 42.07 , in close agreement with the results reported for this atom using a smaller basis set. ${ }^{35}$ The first intracule and extracule shells of Ne correspond to pairs of electrons which are very close in space, or whose center of mass is very close to the atomic nucleus, respectively. These electron pairs are expected to be furnished mainly by core electrons from the 
inner shell in $\rho(\mathbf{r})$. However, one must take into account that the first intracule and extracule shells can also get contributions from core-valence or valence-valence electron pairs, which have a nonzero probability of being at short interelectronic distances, or having the center of mass close to the nucleus. The analysis of the uncorrelated and correlated contributions to the electron-pair shells for the Ne atom (Table II) reveals that electron correlation is important within the two shells. For instance, the correlated contributions to the first and second intracule shells are -1.27 and -3.73 . However, even though the correlated contribution is larger for the second shell, one must take into account that the uncorrelated contributions are 3.68 and 46.33 for the first and second shell, respectively. Thus, the relative importance of the correlated contribution is still higher for the first shell. Similar results are found for the extracule density, where the correlated contributions are -0.93 and -4.08 , and the uncorrelated contributions are 3.86 and 46.15 for the first and second shells, respectively.

When comparing the intracule and extracule shell populations for the $\mathrm{Ne}$ atom, it is found that the uncorrelated contribution to the first shell (3.68) is larger, in absolute value, than the uncorrelated contribution to the first extracule shell (3.86). In contrast, the correlated contribution is larger, in absolute value, for the first intracule shell $(-1.27)$ than for the first extracule shell $(-0.93)$, reflecting a greater importance of Fermi correlation for small values of the intracule coordinate $\mathbf{r}$ than for the extracule coordinate $\mathbf{R}$. All in all, these two trends lead to a significantly larger population for the first extracule shell (2.93) compared to the corresponding intracule shell (2.40). Accordingly, the inverse trend is found for the second intracule and extracule shells.

A similar analysis can be carried out for Ar. The intracule shells for this atom are 1.94, 61.61, and 89.47. The correlated contributions to these shells are $-1.15,-5.02$, and -2.83 , while the uncorrelated contributions are $3.09,66.63$, and 92.30. Thus, the relative importance of electron correlation in the first two shells is similar to that in the Ne atom. In contrast, for the third shell, which corresponds to pairs of electrons with large interelectronic distances, the correlated contribution is small, but nonvanishing. Similar trends are found for the extracule density, with shell populations of $2.50,59.91$, and 90.61 , correlated contributions of -0.76 , -4.12 , and -4.12 , and uncorrelated contributions of 3.26 , 64.03, and 94.72. Following the same trends found for $\mathrm{Ne}$, the correlated contributions are more important for the first intracule than for the first extracule shell of the Ar atom, while the opposite trend is found for the outer intracule and extracule shells, leading to a larger population for the first extracule (2.50) than for the first intracule shell (1.94). The same trend is found for the outer shells (90.61 and 89.47 for the extracule and intracule shell populations) while the inverse is found for the second extracule (59.91) and intracule (61.61) shells.

For all the atoms, CI electron-pair shell populations are very close to those obtained by using the HF approximation (see Table II). For the intracule densities, Coulomb correlation is expected to increase the contribution of $\Gamma^{C}$ into the inner shells. Accordingly, for Ne, there is a slight redistribu- tion of both correlated and uncorrelated electron-pair (e.p.,) densities from the outer to the inner shell $(\sim 0.08$ e.p. in both cases). The redistributions of the uncorrelated and correlated components nearly cancel each other, leading to a final redistribution of total intracule density $\mathrm{Ne}$ of $\sim 0.02$ e.p. from the outer to the inner shell. For Ar, the HF and CI populations of the first shell remain almost constant, but there is some redistribution of the uncorrelated and correlated contributions from the third to the second shell, at the CI level. As for $\mathrm{Ne}$, the uncorrelated and correlated redistributions are very similar in magnitude and the final effect on the total intracule density is very small.

For extracule densities, similar redistributions of shell population are found upon inclusion of Coulomb correlation. For instance, for Ne there is a redistribution of the correlated contribution from the second towards the first shell, while for Ar the redistribution is mainly from the second towards the third shell. In contrast, in both cases there is some redistribution of uncorrelated electron-pair density from the outer to the first (for $\mathrm{Ne}$ ) or second shell (for Ar). Altogether, the final effect is the redistribution of some extracule population ( $\sim 0.03$ and 0.2 e.p., for $\mathrm{Ne}$ and $\mathrm{Ar}$, respectively) from the outer to the second outermost shell.

In summary, the comparison of the $\mathrm{HF}$ and CI results confirms that Fermi correlation accounts for the intracule and extracule shell structure of the $\mathrm{Ne}$ and $\mathrm{Ar}$ atoms, while the effect of Coulomb correlation on the atomic shell structure is small. In terms of shell population, Coulomb correlation leads to a shifting of intracule and extracule population from outer to inner shells.

\section{B. The $\mathrm{C}_{2}^{-2}, \mathrm{~N}_{2}$, and $\mathrm{O}_{2}^{+2}$ series}

Extending the topological analysis of contracted electron-pair densities from atomic to molecular systems is a complex task due to the great number of different electronelectron interactions that can be generated for even the simplest molecules. ${ }^{12,13}$ Therefore, we have considered a series of diatomic homonuclear molecules, $\mathrm{C}_{2}^{-2}, \mathrm{~N}_{2}$, and $\mathrm{O}_{2}^{+2}$, in order to minimize the number of different formal electronelectron interactions, and carry out an initial investigation on the characteristics of the correlated and uncorrelated components of the electron-pair density in small molecules. Moreover, the fact that these three molecules are isoelectronic will allow for a straightforward comparison of the corresponding electron-pair densities. In addition, the effects of increasing the nuclear attraction along this series on the pair density and its components will be analyzed.

In particular, this analysis will be carried out only for $I(\mathbf{r})$ and its components along the molecular axis, for each molecule. Figure 2 collects graphs for $I^{(\mathrm{HF})}, I^{U(\mathrm{HF})}$, and $I^{C(\mathrm{HF})}$, as well as differences between results obtained at the $\mathrm{CI}$ and HF levels. In order to better understand the effect of Coulomb correlation on $I(\mathbf{r}), I^{U}(\mathbf{r})$, and $I^{C}(\mathbf{r})$, CI-HF $\rho(\mathbf{r})$ differences along the molecular axis are also depicted in Fig. 3 for the three molecules.

The topology of $I^{(\mathrm{HF})}$ for $\mathrm{C}_{2}^{-2}, \mathrm{~N}_{2}$, and $\mathrm{O}_{2}^{+2}$ is that expected for diatomic molecules. ${ }^{8,9}$ Thus, for each molecule, there are three peaks of comparable magnitude along the 


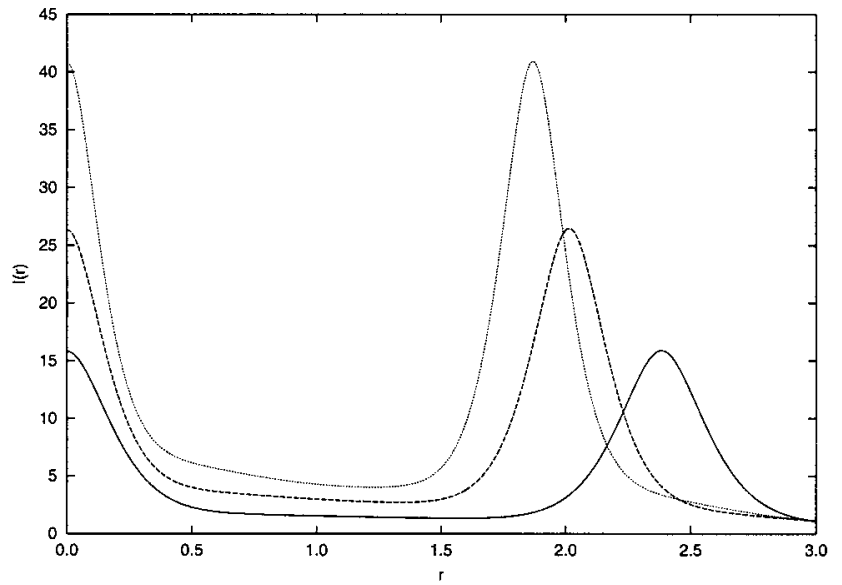

(a)

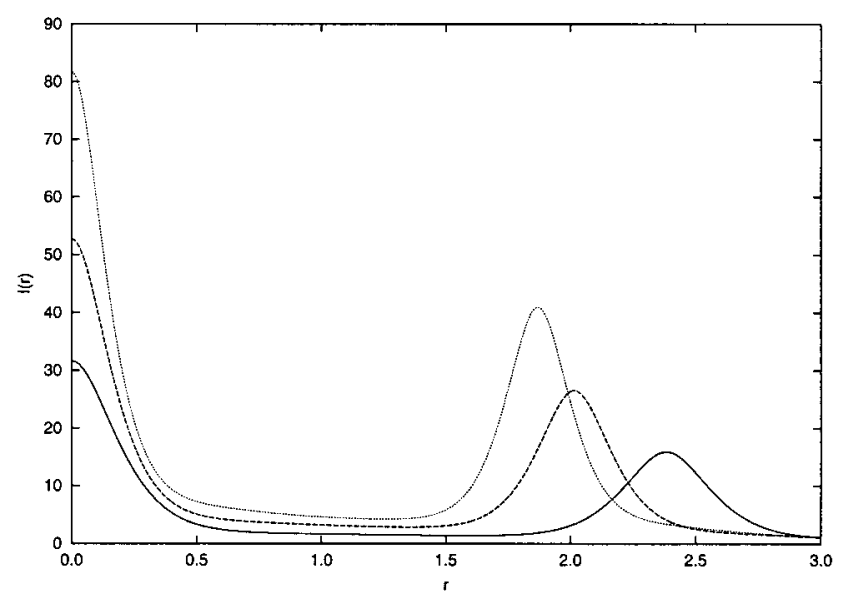

(b)

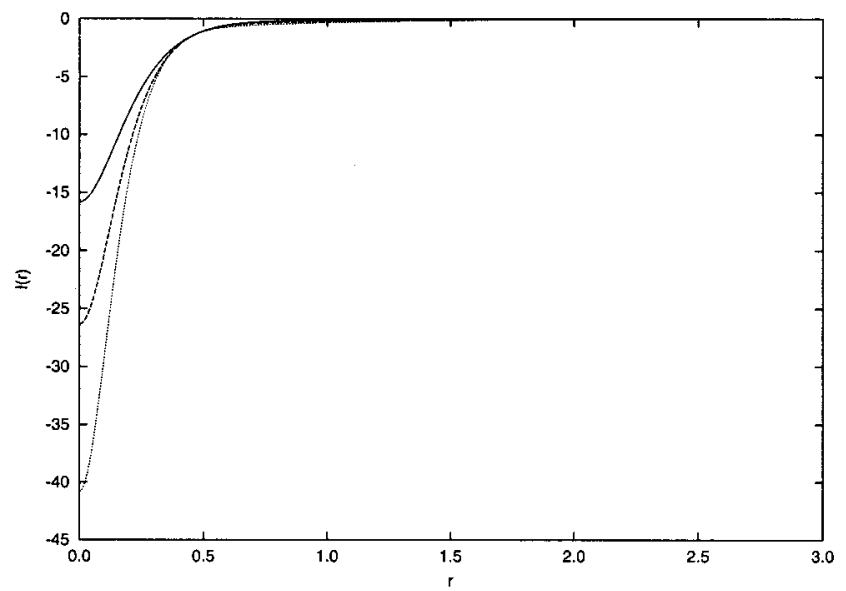

(c)

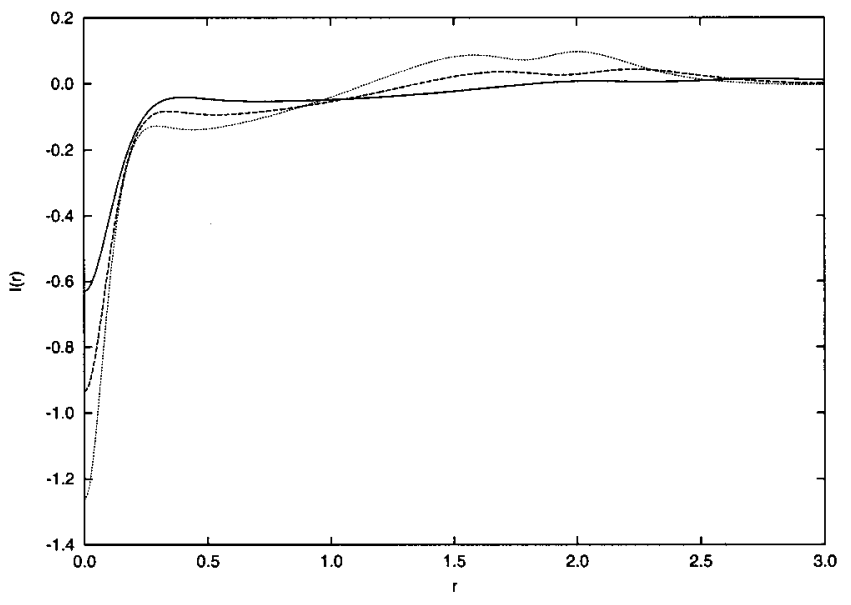

(d)

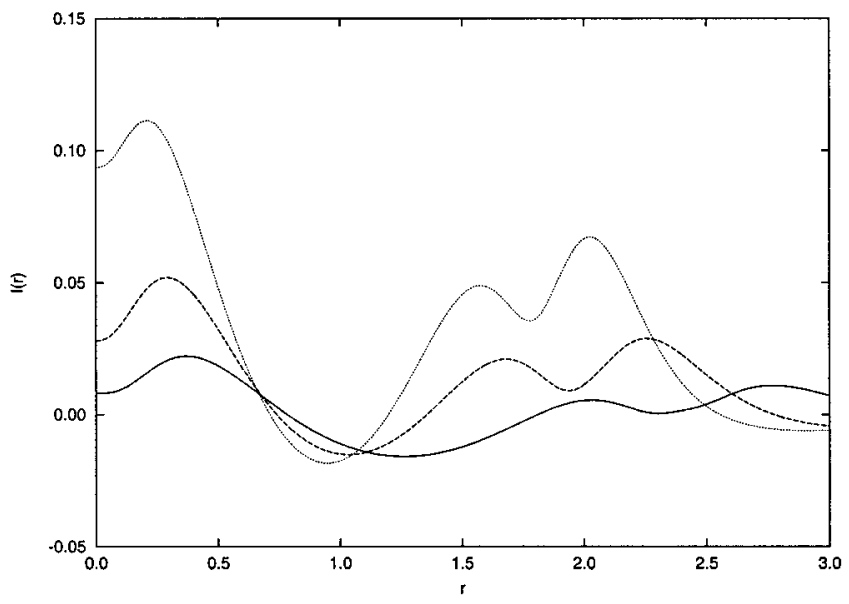

(e)

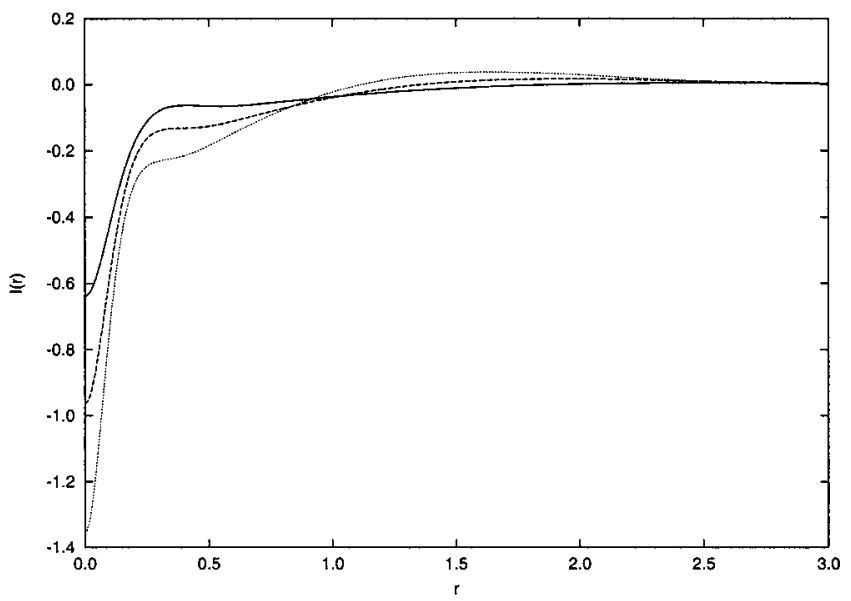

(f)

FIG. 2. Intracule density and its components along the molecular axis for the $\mathrm{C}_{2}^{-2}$ (solid line), $\mathrm{N}_{2}$ (dashed line), and $\mathrm{O}_{2}^{+2}$ (dotted line) molecules. $\mathrm{HF}$ results and CI-HF differences are shown: (a) $I^{(\mathrm{HF})}$, (b) $I^{U(\mathrm{HF})}$, (c) $I^{C(\mathrm{HF})}$, (d) $\Delta I^{(\mathrm{CI}-\mathrm{HF})}$, (e) $\Delta I^{U(\mathrm{CI}-\mathrm{HF})}$, and (f) $\Delta I^{C(\mathrm{CI}-\mathrm{HF})}$.

molecular axis: one at the origin and two symmetric peaks at the positions corresponding approximately to the positive and negative values of the interatomic distance [only one of the symmetric peaks is shown in Fig. 2(a) for each molecule]. The peak at the origin can be related to the contribution of formally intraatomic pairs of electrons, while the other two peaks can be considered to be furnished by inter- atomic electron pairs. ${ }^{8,13}$ The height of all the peaks increases as the nuclear charge increases and the interatomic distance is shortened, along the $\mathrm{C}_{2}^{-2}, \mathrm{~N}_{2}$, and $\mathrm{O}_{2}^{+2}$ series. In fact, the height of the intraatomic peak is the coalescence density $I(0)$ which, for isoelectronic series within the restricted HF aproximation, has been shown to be directly related to a functional of the one-electron density ${ }^{36}$ 


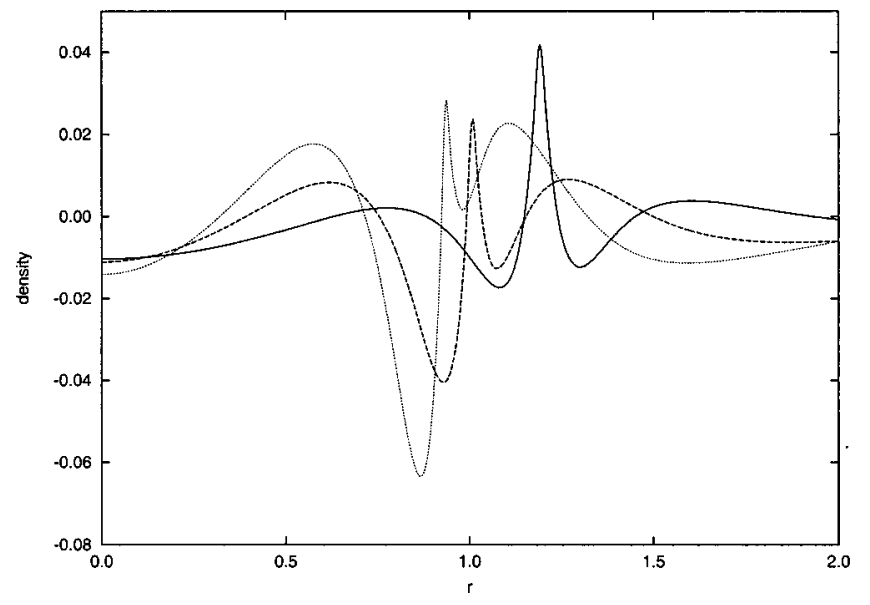

FIG. 3. (CI-HF) $\rho$ (r) differences along the molecular axis for $\mathrm{C}_{2}^{-2}$ (solid line), $\mathrm{N}_{2}$ (dashed line), and $\mathrm{O}_{2}^{+2}$ (dotted line).

$$
I(0)=\frac{\langle\rho\rangle}{4} ; \quad\langle\rho\rangle=\int \rho(\mathbf{r}) \rho(\mathbf{r}) d \mathbf{r} .
$$

$\langle\rho\rangle$ in Eq. (8) has been actually used as a measure of local charge density concentration: the more locally concentrated the one-electron density distribution, the larger the value of $\langle\rho\rangle$ and vice versa. ${ }^{37}$ For instance, $\langle\rho\rangle$ increases along the $\mathrm{C}_{2}^{-2}, \mathrm{~N}_{2}$, and $\mathrm{O}_{2}^{+2}$ series, in agreement with the tighter distribution of charge density around the atoms caused by the increasing nuclear charge, both at the HF and CI levels of theory. ${ }^{38}$

In good agreement with Eq. (8), Fig. 2(a) shows that the increasing concentration of charge density along this series leads to an increasing probability of having electron pairs with short interelectronic distances. The height of the interatomic peaks follows the same trend, revealing that a higher local concentration of charge density around each atom also leads to a more tight distribution of interelectronic distances for formally interatomic electron-electron interactions. Furthermore, similar trends are also found for the intraatomic and interatomic peaks in $I^{U(\mathrm{HF})}$ for the three molecules [see Fig. 2(b)], with the difference that the heights of the intraatomic and interatomic peaks in $I^{U(\mathrm{HF})}$ are not equivalent. On the contrary, the intraatomic peaks at the origin have $I^{U(\mathrm{HF})}$ values that are approximately twice those of the interatomic peaks.

$I^{C(\mathrm{HF})}$ is shown in Fig. 2(c) for the three molecules. It is found that electron (Fermi) correlation is increasingly more important for $\mathrm{C}_{2}^{-2}, \mathrm{~N}_{2}$, and $\mathrm{O}_{2}^{+2}$, again in agreement with the progressive increase in charge density concentration along this series. Furthermore, for the three systems, the range of strong Fermi correlation is found to be small and comparable to that found for isolated atoms [compare the $-I^{C(\mathrm{HF})}$ plot for $\mathrm{Ne}$ in Fig. 1(a) and the $I^{C(\mathrm{HF})}$ plot for $\mathrm{N}_{2}$ in Fig. 2(c)], confirming that there is little Fermi correlation between core electrons located on different atoms. Therefore, exchange or Fermi correlation can be expected to be important only for intraatomic electron-electron interactions and, to a lesser extent, for interatomic interactions between valence electrons from neighboring atoms. ${ }^{21}$
The CI-HF difference graphs [Figs. 2(d), 2(e), and 2(f)] show the effect of an increase of the level of theory on the three molecules. Figure 2(d) reveals that the main differences between $I^{(\mathrm{HF})}$ and $I^{(\mathrm{CI})}$ are found at small values of the intracule coordinate r. However, a slight redistribution of intracule density is also found at larger interelectronic distances ( $\sim 1.5-2.5$ a.u.). For the uncorrelated component [Fig. 2(e)] the main differences between the $\mathrm{HF}$ and $\mathrm{CI}$ results are also found at the origin of the intracule space and at interelectronic distances between 1.5 and 2.5 a.u. Since the topology of $I^{U}(\mathbf{r})$ depends on that of the one-electron density, the $\Delta I^{U(\mathrm{CI}-\mathrm{HF})}$ graph can be interpreted easily in terms of the effects of Coulomb correlation on the one-electron density. Therefore, CI-HF $\rho(\mathbf{r})$ differences along the molecular axis are depicted in Fig. 3 for the three molecules. The three graphs exhibit some common trends. First of all, HF underestimates the value of $\rho(\mathbf{r})$ within a small region around each atomic nucleus, as reflected in the maxima located approximately at the positions of the $\mathrm{C}, \mathrm{N}$, and $\mathrm{O}$ nuclei, respectively, in the $\mathrm{C}_{2}^{-2}, \mathrm{~N}_{2}$, and $\mathrm{O}_{2}^{+2}$ graphs. Figure 3 also reveals that, surrounding each of the atoms, there are two more shells where $\rho(\mathbf{r})$ is overestimated and underestimated, respectively, at the HF level of theory. Note that the density at the bond critical point (the origin of coordinates in Fig. 3) is systematically overestimated at the HF level. These trends have an immediate translation in terms of $\Delta I^{U(\mathrm{CI}-\mathrm{HF})}$. First of all, the tighter concentration of charge density around each atom at the CI level, with respect to the HF approximation, leads to a corresponding increase of the probability of having uncorrelated electrons close to each other, as reflected in the region around the origin in Fig. 2(e). Accordingly, the probability for uncorrelated electron-electron interactions with interelectronic distances close to the interatomic distance also increases with the level of theory [see Fig. 2(e)]. As expected, the magnitude of the $\mathrm{CI}-\mathrm{HF}$ correlation shifts on $I^{U}(\mathbf{r})$ and $\rho(\mathbf{r})$ increases along the $\mathrm{C}_{2}^{-2}, \mathrm{~N}_{2}$, and $\mathrm{O}_{2}^{+2}$ series.

Finally, the difference plot for the correlated component $\Delta I^{C(\mathrm{CI}-\mathrm{HF})}$ confirms that considering Coulomb correlation increases the depth of the Fermi or exchange-correlation hole for small interelectronic distances. As for Fermi correlation, it is found that Coulomb correlation becomes more important with the increasing local concentration of the one-electron density along the $\mathrm{C}_{2}^{-2}, \mathrm{~N}_{2}$, and $\mathrm{O}_{2}^{+2}$ series. A comparison of $\Delta I^{U(\mathrm{CI}-\mathrm{HF})}$ and $\Delta I^{C(\mathrm{CI}-\mathrm{HF})}$ [Figs. 2(e) and 2(f), respectively] shows that the correlation shift is about one order of magnitude larger for $\Gamma^{C}$ than for $\Gamma^{U}$.

In summary, the results depicted in Figs. 2(d), 2(e), and 2(f) reveal that introducing Coulomb correlation at the CI level works to produce two apparently contrary effects on the intracule density. First, for this series of molecules, the CI one-electron density is more compact and locally concentrated around the atomic nuclei than the corresponding $\mathrm{HF}$ density, leading to a slight increase in the probability for short distance uncorrelated electron-electron interactions, as revealed by the $\Delta I^{U(\mathrm{CI}-\mathrm{HF})}$ plot in Fig. 2(e). However, taking into account Coulomb correlation also increases the depth of the HF Fermi correlation hole $\left[\right.$ see $\Delta I^{C(\mathrm{CI}-\mathrm{HF})}$ plots in Fig. 2(f)]. Since the electron-pair redistribution on $\Gamma^{C}$ is quantitatively more important than on $\Gamma^{U}$, one finally obtains a 
smaller probability for short distance electron-electron interactions at the CI level. Thus, at the same time that electrons are kept more locally concentrated around atomic nuclei, they are also kept at larger interelectronic distances.

\section{The $\mathrm{C}_{2} \mathrm{H}_{4}$ molecule}

The $\mathrm{C}_{2} \mathrm{H}_{4}$ molecule will be considered for the application of the analysis described above to a polyatomic molecule. Because of the high symmetry of this molecule, the number of nonequivalent electron-electron interactions is quite low, simplifying the topological analysis of the intracule and extracule densities. Moreover, the topologies of the $I(\mathbf{r}), E(\mathbf{R}), \nabla^{2} I(\mathbf{r})$, and $\nabla^{2} E(\mathbf{R})$ distributions of the $\mathrm{C}_{2} \mathrm{H}_{4}$ molecule have been recently investigated at the HF/6-31G* level of theory. ${ }^{13}$ While very few electron-electron interactions could be associated to local maxima in the $I(\mathbf{r})$ and $E(\mathbf{R})$ densities, most interactions could be associated to local minima in the corresponding Laplacian functions. Therefore, the analysis of the $\mathrm{C}_{2} \mathrm{H}_{4}$ molecule has been restricted to the $\nabla^{2} I(\mathbf{r})$ and $\nabla^{2} E(\mathbf{R})$ distributions and its components, calculated at the HF and CI levels of theory.

Intracule and extracule contour maps of $\mathrm{C}_{2} \mathrm{H}_{4}$ are presented in Figs. 4 and 5, respectively. All the maps lie on the plane defined by the atoms in the molecule, with the carbon atoms located along the $x$ axis. In turn, Table III reports the local minima characterized in $\nabla^{2} I^{(\mathrm{HF})}, \nabla^{2} I^{(\mathrm{CI})}, \nabla^{2} I^{U(\mathrm{HF})}$, and $\nabla^{2} I^{U(\mathrm{Cl})}$, as well as maxima characterized in $\nabla^{2} I^{C(\mathrm{HF})}$ and $\nabla^{2} I^{C(\mathrm{Cl})}$. Accordingly, local minima characterized in $\nabla^{2} E^{(\mathrm{HF})}, \nabla^{2} E^{(\mathrm{HF})}, \nabla^{2} E^{U(\mathrm{CI})}$, and $\nabla^{2} E^{U(\mathrm{Cl})}$, and maxima characterized in $\nabla^{2} E^{C(\mathrm{HF})}$ and $\nabla^{2} E^{C(\mathrm{CI})}$ are collected in Table IV. For the labeling of the electron-electron interactions in $\mathrm{C}_{2} \mathrm{H}_{4}$, the same notation introduced in Ref. 13 is used. Thus, $\left\{\mathrm{C}_{i i}\right\}$ and $\left\{\mathrm{H}_{i i}\right\}$ refer to intraatomic electronelectron interactions on $\mathrm{C}$ and $\mathrm{H}$, respectively, while $\left\{\mathrm{C}_{i} \mathrm{C}_{j}\right\}$, $\left\{\mathrm{C}_{i} \mathrm{H}_{i}\right\},\left\{\mathrm{C}_{i} \mathrm{H}_{j}\right\},\left\{\mathrm{H}_{i} \mathrm{H}_{i}\right\}$, and $\left\{\mathrm{H}_{i} \mathrm{H}_{j}\right\}$ refer to different kinds of interatomic interactions. $i i$ is used to label interactions between electrons belonging to atoms from the same $\mathrm{CH}_{2}$ group, while $i j$ is used to label interactions between electrons on atoms from different $\mathrm{CH}_{2}$ groups. $\left\{\mathrm{H}_{i} \mathrm{H}_{j}\right\}$ interactions are divided into cis and trans. Finally, $\{0\}$ is used to label sets of interactions contributing collectively to the origin of coordinates in the intracule or extracule distributions. Because of the molecular symmetry of $\mathrm{C}_{2} \mathrm{H}_{4}$, only the positive quadrant of each map needs to be discussed.

Figures 4(a) and 4(b) depict the $\nabla^{2} I^{(\mathrm{HF})}$ and $\nabla^{2} I^{(\mathrm{CI})}$ distributions, which are topologically equivalent. From a quantitative point of view, there are two interactions whose $I(\mathbf{r})$ values change significantly depending on the approximation used. First, the intracule density at the origin, associated to the interaction $\{0\}$, decreases from a value of 15.847 at the HF level to 15.715 at the CI level (see Table III). Second, the $I(\mathbf{r})$ value associated to the $\left\{C_{i} C_{j}\right\}$ interaction is 0.018 a.u. larger at the CI level. Local minima associated to the rest of the interactions change only slightly at the HF and CI levels of theory. Also, with respect to CI, HF underestimates by $\sim 0.013$ a.u. the interelectronic distance associated to the $\left\{\mathrm{H}_{i} \mathrm{H}_{j}\right\}$ cis and trans interactions.
The $\nabla^{2} I^{U(\mathrm{HF})}$ and $\nabla^{2} I^{U(\mathrm{Cl})}$ distributions of $\mathrm{C}_{2} \mathrm{H}_{4}$ are topologically equivalent between them, and also to the $\nabla^{2} I^{(\mathrm{HF})}$ and $\nabla^{2} I^{(\mathrm{CI})}$ distributions. Therefore, only $\nabla^{2} I^{U(\mathrm{CI})}$ is depicted [Fig. 4(c)]. From a quantitative point of view, the main differences between $\nabla^{2} I^{(\mathrm{Cl})}$ and $\nabla^{2} I^{U(\mathrm{Cl})}$, and between $\nabla^{2} I^{(\mathrm{HF})}$ and $\nabla^{2} I^{U(\mathrm{HF})}$, are found for the minima associated to the $\{0\}$ interaction, at all levels of theory (see Table III). In agreement with the relationship found for the intracule density at the origin for the atomic series, $I(0)$ is equal to $I^{U}(0) / 2$ at the HF level and smaller at the CI level. For the rest of the electron-electron interactions, the differences between the minima in $\nabla^{2} I^{(\mathrm{HF})}$ and $\nabla^{2} I^{U(\mathrm{HF})}$ are small, especially for long-distance interactions, and the same trend is found for $\nabla^{2} I^{(\mathrm{CI})}$ and $\nabla^{2} I^{U(\mathrm{CI})}$.

The comparison of the $\nabla^{2} I(\mathbf{r})$ and $\nabla^{2} I^{U}(\mathbf{r})$ distributions above suggests that the overall topology of molecular $\nabla^{2} I(\mathbf{r})$ distributions is determined mainly by the uncorrelated component, $\nabla^{2} I^{U}(\mathbf{r})$. Thus, the correlated component $\nabla^{2} I^{C}(\mathbf{r})$ can be expected to have only a relatively small contribution to the topology of the total $\nabla^{2} I(\mathbf{r})$ distribution. However, $\nabla^{2} I^{C}(\mathbf{r})$ is important, from a chemical point of view, because it reflects the way in which the electrons in the system are correlated. Moreover, $I^{C}(\mathbf{r})$ has been found to be especially sensible to the level of theory for the atomic and molecular series discussed above. In order to assess the effects of Fermi and Coulomb correlation on molecular $-\nabla^{2} I^{C}(\mathbf{r})$ distributions, three different maps are presented. Figures $4(\mathrm{~d})$ and $4(\mathrm{e})$ show the $-\nabla^{2} I^{C(\mathrm{HF})}$ and $-\nabla^{2} I^{C(\mathrm{CI})}$ maps of $\mathrm{C}_{2} \mathrm{H}_{4}$, respectively, while Fig. 4(f) depicts the $-\nabla^{2} \Delta I^{C(\mathrm{CI}-\mathrm{HF})}$ difference map. Note that the functions represented in Figs. 4(d), 4(e), and 4(f) correspond in fact to the Laplacians of the anisotropic Fermi, exchange correlation and Coulomb holes, respectively. Note also that, for the sake of comparison with $\nabla^{2} I(\mathbf{r})$ and $\nabla^{2} I^{U}(\mathbf{r})$ maps, $-\nabla^{2} I^{C}(\mathbf{r})$ maps have been depicted instead of $\nabla^{2} I^{C}(\mathbf{r})$ maps, in Figs. 4(d), 4(e), and 4(f). Thus, taking into account that $I^{C}(\mathbf{r})$ is negative for any value of $\mathbf{r}$, negative and positive values in these maps correspond to local $\left|I^{C}(\mathbf{r})\right|$ concentration and depletion, respectively.

The main topological feature in the $-\nabla^{2} I^{C(\mathrm{HF})}$ distribution [Fig. 4(d)] is a local minimum at the origin, surrounded by two shells of positive and negative Laplacian values, respectively, the latter one being extended up to $\sim 2$ a.u. from the origin. Within this region, $-\nabla^{2} I^{C(\mathrm{HF})}$ is nearly isotropic on the $x z$ plane. At larger interelectronic distances, local minima can be assigned to the $\left\{\mathrm{C}_{i} \mathrm{C}_{j}\right\},\left\{\mathrm{C}_{i} \mathrm{H}_{i}\right\}$, and $\left\{\mathrm{H}_{i} \mathrm{H}_{j}\right\}$ (cis and trans) interactions, but no minima can be associated to the $\left\{\mathrm{C}_{i} \mathrm{H}_{j}\right\}$ and $\left\{\mathrm{H}_{i} \mathrm{H}_{i}\right\}$ interactions. According to the $I^{C}(\mathbf{r})$ values reported in Table III, the degree of Fermi correlation associated to different core-core electron interactions decreases in the order $\left\{\mathrm{C}_{i} \mathrm{H}_{i}\right\},\left\{\mathrm{C}_{i} \mathrm{C}_{j}\right\},\left\{\mathrm{H}_{i} \mathrm{H}_{j}\right\}$ (cis), and $\left\{\mathrm{H}_{i} \mathrm{H}_{j}\right\}$ (trans).

The $-\nabla^{2} I^{C(\mathrm{HF})}$ and $-\nabla^{2} I^{C(\mathrm{CI})}$ maps [Figs. 4(d) and 4(e)] are very similar. In both cases the same set of interactions are reflected as local minima, except for the $\left\{\mathrm{C}_{i} \mathrm{H}_{i}\right\}$ and $\left\{\mathrm{C}_{i} \mathrm{H}_{j}\right\}$ interactions, which are characterized only at the HF and CI levels of theory, respectively. From a quantitative point of view, the main differences are found in the minima associated to the $\{0\}$ and $\left\{\mathrm{C}_{i} \mathrm{C}_{j}\right\}$ interactions. The $I^{C}(0)$ value goes from -15.847 at the $\mathrm{HF}$ level to -15.995 at the 


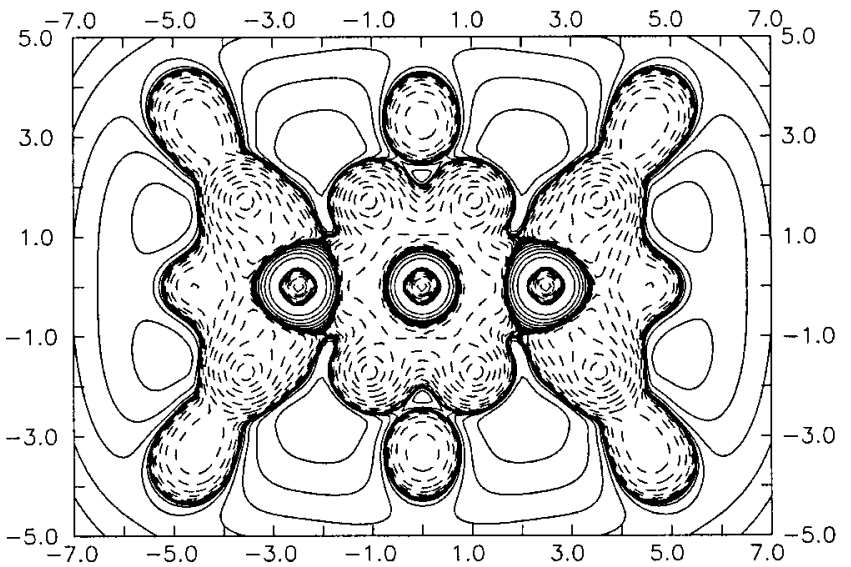

(a)

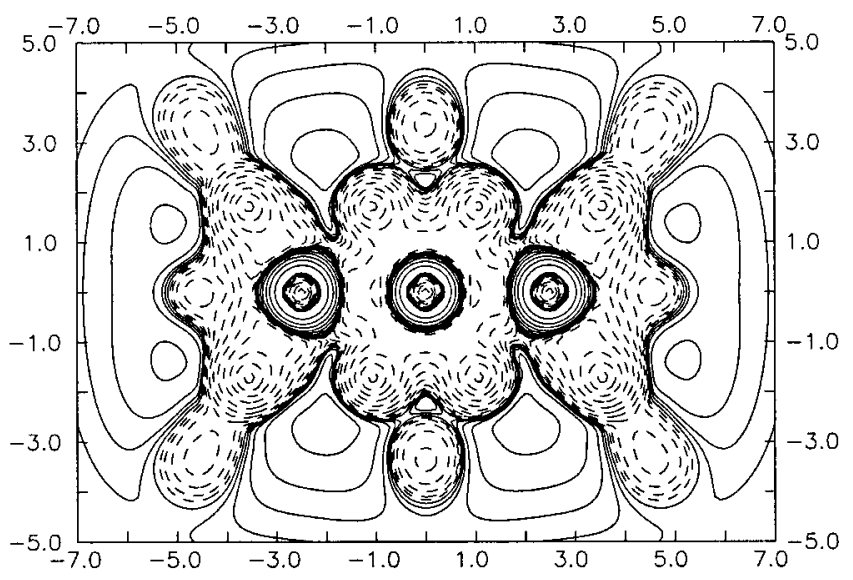

(b)

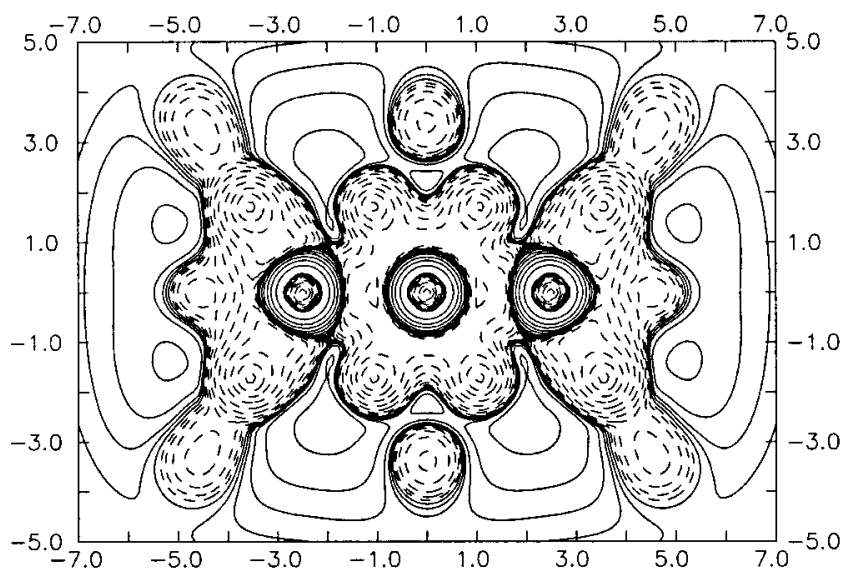

(c)

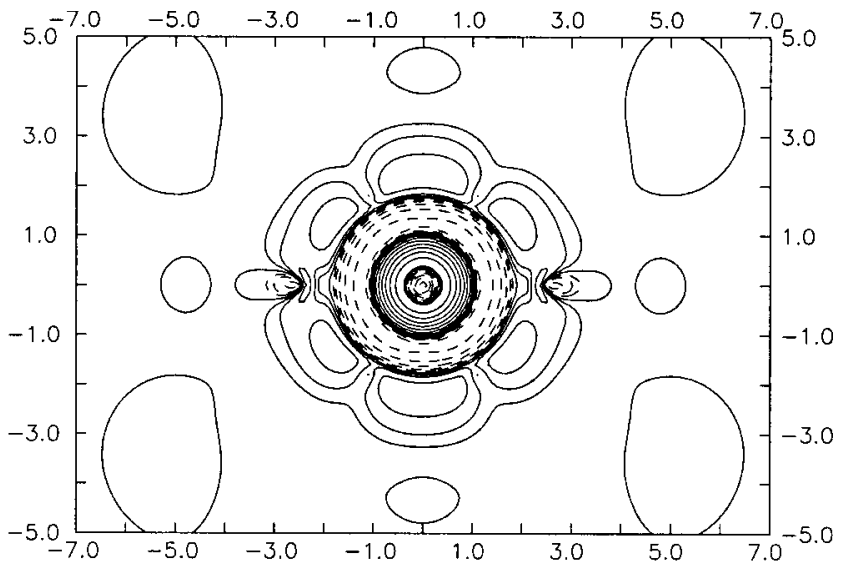

(d)

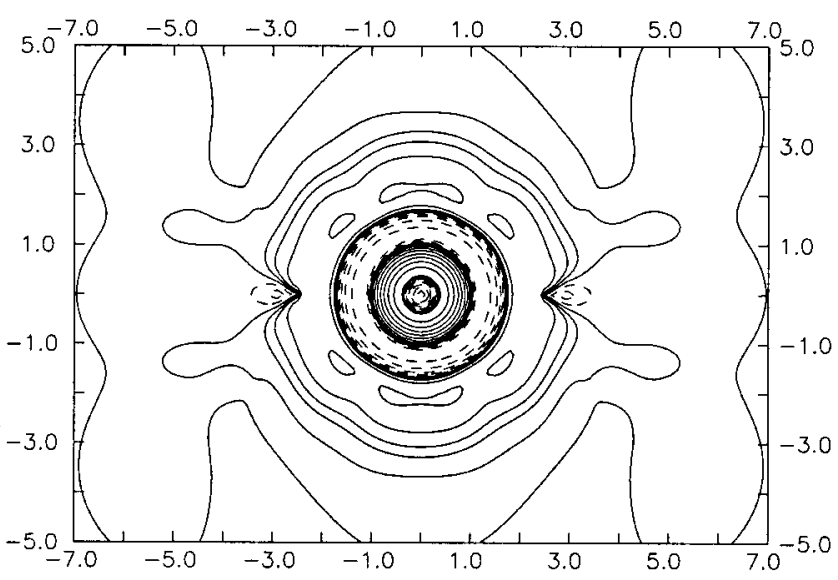

(e)

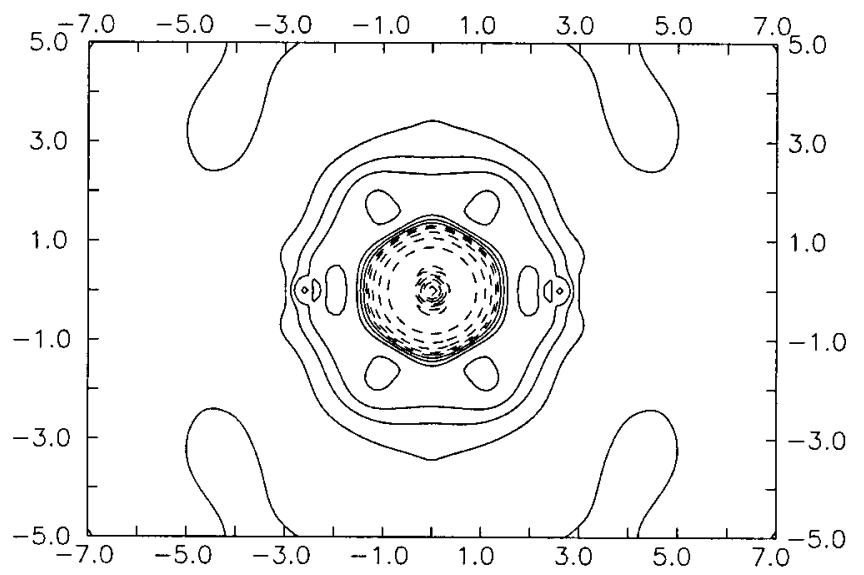

(f)

FIG. 4. Intracule contour maps for the $\mathrm{C}_{2} \mathrm{H}_{4}$ molecule. Positive values are depicted in solid lines and negative values in dashed lines: (a) $\nabla^{2} I^{(\mathrm{HF})}$ map, in contours of $0.01 \times 2^{n}$ a.u.; (b) $\nabla^{2} I^{(\mathrm{Cl})}$ map, in contours of $0.01 \times 2^{n}$ a.u.; (c) $\nabla^{2} I^{U(\mathrm{Cl})}$ map, in contours of $0.01 \times 2^{n}$ a.u.; (d) $-\nabla^{2} I^{C(\mathrm{HF})}$ map, in contours of $0.01 \times 2^{n}$ a.u.; (e) $-\nabla^{2} I^{C(\mathrm{Cl})}$ map, in contours of $0.01 \times 2^{n}$ a.u.; and (f) $-\Delta \nabla^{2} I^{C(\mathrm{CI}-\mathrm{HF})}$ map, in contours of $0.01 \times 2^{n}$ a.u.

CI level (see Table III), reflecting that the correlation hole is slightly deeper when taking into account Coulomb correlation. In turn, the $\mathrm{HF}$ and $\mathrm{CI} I^{C}(\mathbf{r})$ values associated to the $\left\{\mathrm{C}_{i} \mathrm{C}_{j}\right\}$ interaction are -0.019 and -0.013 , reflecting a slight overestimation of the correlation associated to this interaction at the HF level of theory.

Within the approach used in this work, at post-HF levels of theory, Fermi and Coulomb correlation contribute to- 


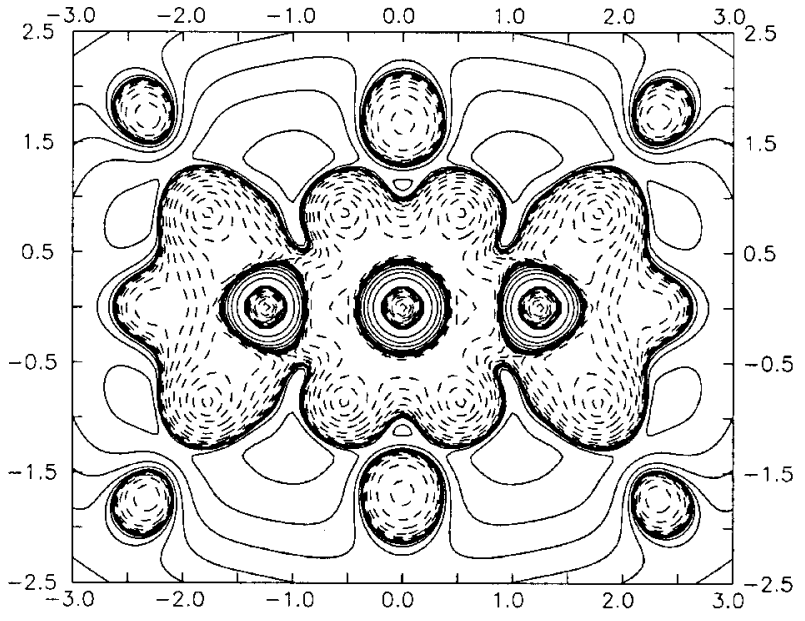

(a)

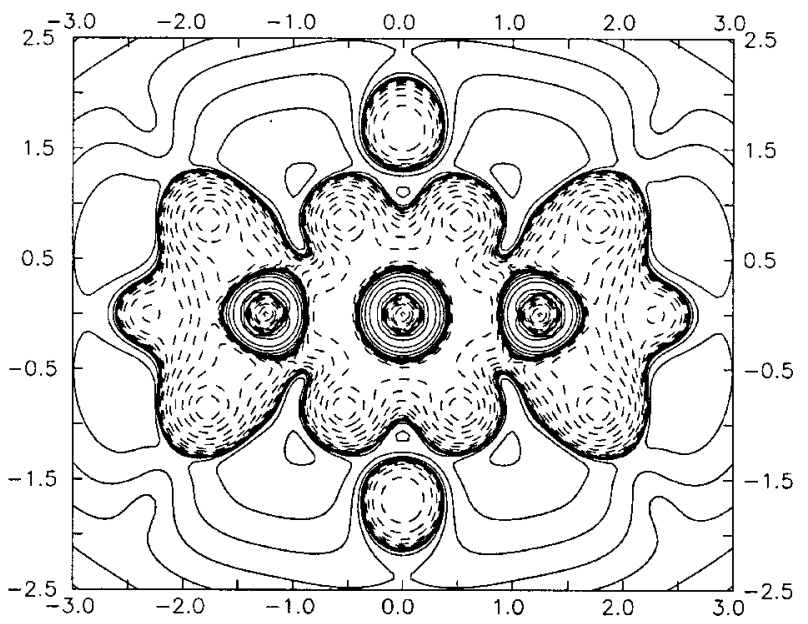

(b)

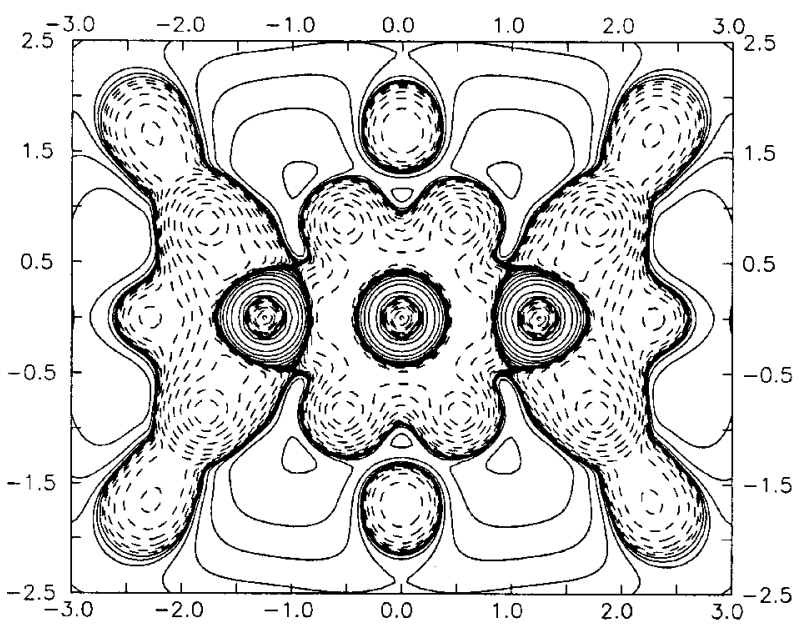

(c)

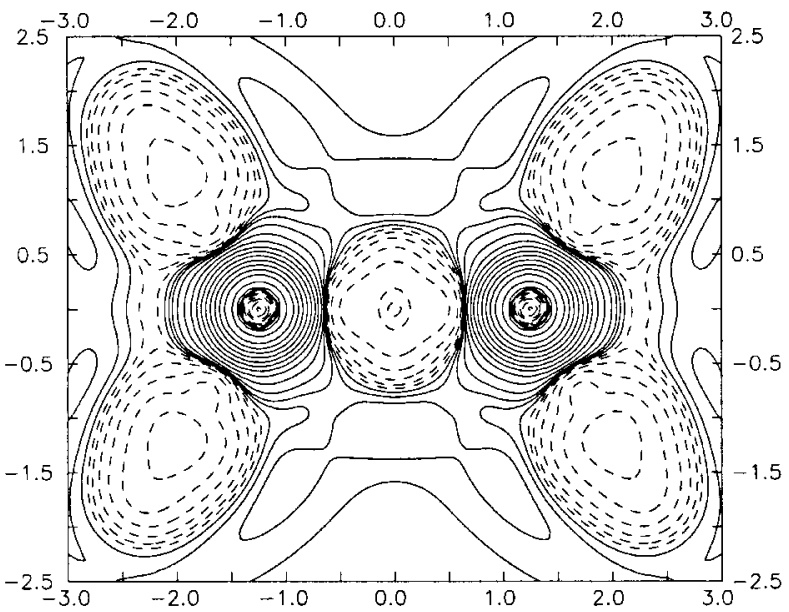

(d)

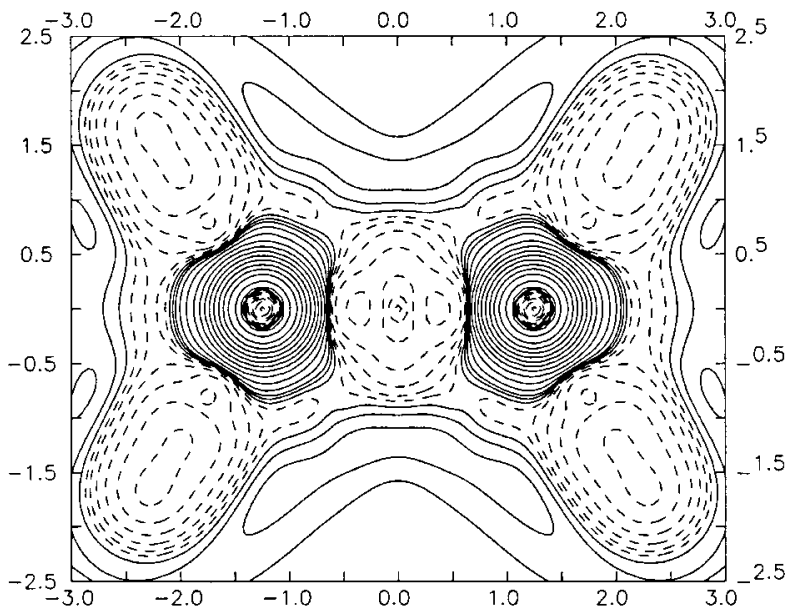

(e)

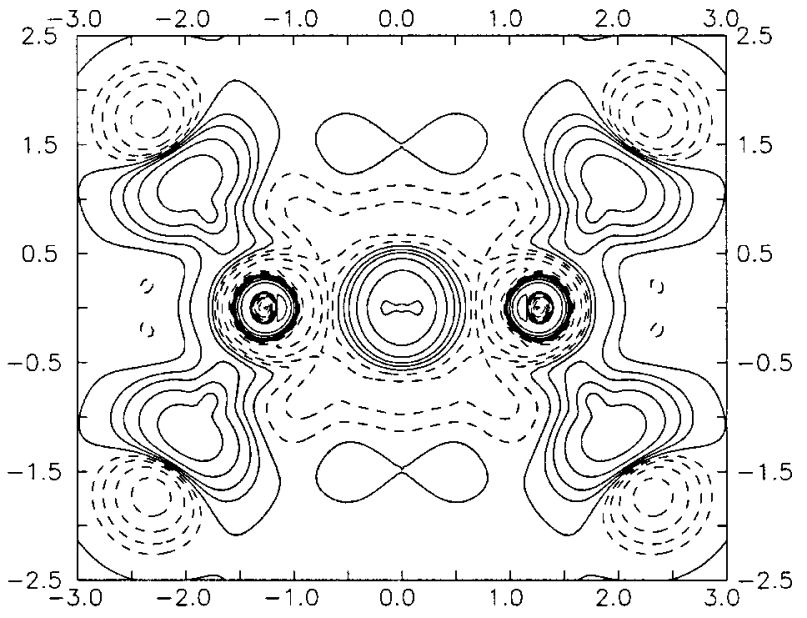

(f)

FIG. 5. Extracule contour maps for the $\mathrm{C}_{2} \mathrm{H}_{4}$ molecule. Positive values are depicted in solid lines and negative values in dashed lines: (a) $\nabla^{2} E^{(\mathrm{HF})}$ map, in contours of $0.1 \times 2^{n}$ a.u.; (b) $\nabla^{2} E^{(\mathrm{Cl})}$ map, in contours of $0.1 \times 2^{n}$ a.u.; (c) $\nabla^{2} E^{U(\mathrm{Cl})}$ map, in contours of $0.1 \times 2^{n}$ a.u.; (d) $-\nabla^{2} E^{C(\mathrm{HF})}$ map, in contours of $0.1 \times 2^{n}$ a.u.; (e) $-\nabla^{2} E^{C(\mathrm{Cl})}$ map, in contours of $0.1 \times 2^{n}$ a.u.; and (f) $-\Delta \nabla^{2} E^{C(\mathrm{CI}-\mathrm{HF})}$ map, in contours of $0.1 \times 2^{n}$ a.u. 
TABLE III. Internuclear distances $\left(\left|\mathbf{D}_{A B}\right|\right)$, interelectronic distances $\left(\left|\mathbf{r}_{a b}\right|\right)$, and intracule densities $[I(\mathbf{r})$, $I^{U}(\mathbf{r})$ and $\left.I^{C}(\mathbf{r})\right]$ of the different electron-electron interactions assigned to local minima in $\nabla^{2} I(\mathbf{r}), \nabla^{2} I^{U}(\mathbf{r})$, and $\nabla^{2} I^{C}(\mathbf{r})$ for the $\mathrm{C}_{2} \mathrm{H}_{4}$ molecule calculated at the $\mathrm{HF}$ (values in roman type) and $\mathrm{CI}$ (values in italics) levels of theory (a.u. are units used throughout).

\begin{tabular}{lccccccc}
\hline \hline & \multicolumn{5}{c}{ Interaction } \\
\cline { 2 - 8 } Intracule & \multicolumn{1}{c}{0} & $\left\{\mathrm{C}_{i} \mathrm{C}_{j}\right\}$ & $\left\{\mathrm{C}_{i} \mathrm{H}_{i}\right\}$ & $\left\{\mathrm{C}_{i} \mathrm{H}_{j}\right\}$ & $\left\{\mathrm{H}_{i} \mathrm{H}_{i}\right\}$ & $\left\{\mathrm{H}_{i} \mathrm{H}_{j}\right\}_{\text {cis }}$ & $\left\{\mathrm{H}_{i} \mathrm{H}_{j}\right\}_{\text {trans }}$ \\
\hline $\mathbf{D}_{A B} \mid$ & $\ldots$ & 2.489 & 2.033 & 3.958 & 3.456 & 4.632 & 5.780 \\
Total & & & & & & & \\
$\left|\mathbf{r}_{a b}\right|$ & 0.000 & 2.489 & 2.018 & 3.945 & 3.369 & 4.579 & 5.653 \\
& 0.000 & 2.489 & 2.019 & 3.946 & 3.376 & 4.593 & 5.666 \\
$I(\mathbf{r})$ & 15.847 & 15.869 & 1.047 & 0.858 & 0.177 & 0.267 & 0.082 \\
& 15.715 & 15.887 & 1.052 & 0.858 & 0.176 & 0.263 & 0.085 \\
Uncorrelated & & & & & & & \\
$\left|\mathbf{r}_{a b}\right|$ & 0.000 & 2.489 & 2.018 & 3.945 & 3.387 & 4.580 & 5.655 \\
& 0.000 & 2.489 & 2.019 & 3.946 & 3.397 & 4.592 & 5.788 \\
$I^{U}(\mathbf{r})$ & 31.694 & 15.891 & 1.095 & 0.860 & 0.177 & 0.268 & 0.082 \\
& 31.709 & 15.901 & 1.095 & 0.859 & 0.174 & 0.264 & 0.081 \\
Correlated & & & & & & & \\
$\left|\mathbf{r}_{a b}\right|$ & 0.000 & 2.584 & 2.163 & $\ldots$ & $\ldots$ & 4.137 & 5.783 \\
$I^{C}(\mathbf{r})$ & 0.000 & 2.563 & $\ldots$ & 4.119 & $\ldots$ & $\ldots$ & 5.891 \\
& -15.847 & -0.019 & -0.037 & $\ldots$ & $\ldots$ & $-1.57 \times 10^{-3}$ & $-0.37 \times 10^{-3}$ \\
& -15.995 & -0.013 & $\ldots$ & $-0.91 \times 10^{-3}$ & $\ldots$ & $\ldots$ & $-0.35 \times 10^{-3}$ \\
\hline \hline
\end{tabular}

gether to the exchange-correlation density. One way to isolate the contribution of Coulomb correlation is to analyze difference maps between contracted electron-pair densities calculated with the HF and CISD approximations. These kind of difference maps can be seen as a generalization of the Coulomb hole, originally defined as the difference between the radial intracule densities calculated with a given $a b$ initio method and with the HF approximation. ${ }^{10}$ The effect of Coulomb correlation can be better appreciated in the
Laplacian difference maps. For instance, the $-\nabla^{2} \Delta I^{C(\mathrm{CI}-\mathrm{HF})}$ map [Fig. 4(f)] shows that CI shifts the correlation hole towards smaller electron-electron distances. This is reflected as a negative region around the origin in $-\nabla^{2} \Delta I^{C(\mathrm{CI}-\mathrm{HF})}$, extended up to interelectronic distances of $\sim 1.5$ a.u. In contrast to the $-\nabla^{2} I^{C(\mathrm{HF})}$ and $-\nabla^{2} I^{C(\mathrm{CI})}$ maps, no shell structure is found in $-\nabla^{2} \Delta I^{C(\mathrm{CI}-\mathrm{HF})}$. From a quantitative point of view, Coulomb correlation is less important than Fermi cor-

TABLE IV. Internuclear distances $\left(\left|\mathbf{D}_{A B}\right|\right)$, interelectronic distances $\left(\left|\mathbf{r}_{a b}\right|\right)$, and extracule densities $[E(\mathbf{R})$, $E^{U}(\mathbf{r})$, and $\left.E^{C}(\mathbf{r})\right]$ off the different electron-electron interactions assigned to local maxima in $\nabla^{2} E(\mathbf{R})$, $\nabla^{2} E^{U}(\mathbf{R})$, and $\nabla^{2} E^{U}(\mathbf{R})$ for the $\mathrm{C}_{2} \mathrm{H}_{4}$ molecule calculated at the $\mathrm{HF}$ (values in roman type) and $\mathrm{CI}$ (values in italics) levels of theory (a.u. are units used throughout).

\begin{tabular}{|c|c|c|c|c|c|c|c|}
\hline \multirow[b]{2}{*}{ Extracule } & \multicolumn{7}{|c|}{ Interaction } \\
\hline & $\{0\}$ & $\left\{\mathrm{C}_{i i}\right\}$ & $\left\{\mathrm{C}_{i} \mathrm{H}_{i}\right\}$ & $\left\{\mathrm{C}_{i} \mathrm{H}_{j}\right\}$ & $\left\{\mathrm{H}_{i i}\right\}$ & $\left\{\mathrm{H}_{i} \mathrm{H}_{i}\right\}$ & $\left\{\mathrm{H}_{i} \mathrm{H}_{j}\right\}_{c i s}$ \\
\hline$\left|\mathbf{D}_{A B}\right|$ & $\cdots$ & 1.244 & 1.979 & 1.016 & 2.890 & 2.316 & 1.728 \\
\hline \multicolumn{8}{|l|}{ Total } \\
\hline \multirow[t]{2}{*}{$\left|\mathbf{r}_{a b}\right|$} & 0.000 & 1.244 & 1.972 & 1.009 & 2.982 & 2.292 & 1.692 \\
\hline & 0.000 & 1.244 & 1.973 & 1.010 & 2.944 & 2.298 & 1.697 \\
\hline \multirow[t]{2}{*}{$E(\mathbf{r})$} & 253.160 & 66.931 & 6.619 & 8.672 & 0.350 & 2.051 & 1.410 \\
\hline & 253.376 & 66.864 & 6.651 & 8.662 & 0.279 & 2.010 & 1.380 \\
\hline \multicolumn{8}{|c|}{ Uncorrelated } \\
\hline \multirow[t]{2}{*}{$\left|\mathbf{r}_{a b}\right|$} & 0.000 & 1.244 & 1.972 & 1.009 & 2.827 & 2.290 & 1.693 \\
\hline & 0.000 & 1.244 & 1.973 & 1.010 & 2.834 & 2.296 & 1.699 \\
\hline \multirow[t]{2}{*}{$E^{U}(\mathbf{r})$} & 253.566 & 127.136 & 6.881 & 8.764 & 0.656 & 2.146 & 1.418 \\
\hline & 253.685 & 127.216 & 6.876 & 8.762 & 0.644 & 2.112 & 1.390 \\
\hline \multicolumn{8}{|l|}{ Correlated } \\
\hline \multirow[t]{2}{*}{$\left|\mathbf{r}_{a b}\right|$} & 0.000 & 1.244 & 1.948 & $\cdots$ & $\cdots$ & 2.253 & $\cdots$ \\
\hline & 0.000 & 1.244 & 1.919 & $\cdots$ & 2.814 & 2.269 & $\cdots$ \\
\hline \multirow[t]{2}{*}{$E^{C}(\mathbf{r})$} & -0.406 & -60.205 & -0.252 & $\cdots$ & $\ldots$ & -0.089 & $\cdots$ \\
\hline & -0.309 & -60.353 & -0.212 & $\cdots$ & -0.259 & -0.098 & $\cdots$ \\
\hline
\end{tabular}


relation. For instance, $I^{C}(0)$ is -15.847 at the HF level of theory, while the difference between the CI and HF $I^{C}(0)$ values is only 0.147 .

We prceed now to discuss the extracule maps, depicted in Fig. 5. As for $\nabla^{2} I^{(\mathrm{HF})}$ and $\nabla^{2} I^{(\mathrm{CI})}$, the $\nabla^{2} E^{(\mathrm{HF})}$ and $\nabla^{2} E^{(\mathrm{CI})}$ distributions are topologically equivalent [Figs. 5(a) and 5(b), respectively]. The comparison between Figs. 5(a) and 5(b) shows that consideration of Coulomb correlation has a significant effect on the $\left\{\mathrm{H}_{i i}\right\}$ interaction. The four equivalent minima associated to this interaction have $E(\mathbf{R})$ values of 0.350 and 0.279 at the HF and CI levels of theory, respectively. Moreover, while at the HF level, the $\nabla^{2} E(\mathbf{R})$ value associated to this interaction is negative $(-0.957)$, it is positive at the CI level (0.309). Thus, these minima are not visually apparent in the $\nabla^{2} E^{(\mathrm{CI})}$ contour map [Fig. 5(b)]. In a valence-bond (VB) language, the fact that these minima are stronger at the HF level is a consequence of the overestimation of the contribution of a set of ionic structures, formally represented as $\mathrm{H}^{-}$, to the molecular wave function. Since the importance of the $\left\{\mathrm{H}_{i i}\right\}$ interaction is related to the weight of these $\mathrm{H}^{-}$VB structures, the corresponding local minima in $\nabla^{2} E^{(\mathrm{HF})}$ are also exaggerated.

The $\nabla^{2} E^{U(\mathrm{Cl})}$ distribution [Fig. 5(c)] exhibits the same topological features found in $\nabla^{2} E^{(\mathrm{CI})}$. As expected, the main differences are found for the intraatomic interactions $\left\{\mathrm{C}_{i i}\right\}$ and $\left\{\mathrm{H}_{i i}\right\}$, which are more important in the uncorrelated map. Similar topological features and quantitative trends are found for $\nabla^{2} E^{U(\mathrm{HF})}$ and $\nabla^{2} E^{U(\mathrm{CI})}$ (see Table IV).

Finally, the effect of Fermi and Coulomb correlation on the extracule distributions can be discussed by means of the $-\nabla^{2} E^{C(\mathrm{HF})},-\nabla^{2} E^{C(\mathrm{CI})}$, and $-\nabla^{2} E^{C(\mathrm{CI}-\mathrm{HF})}$ maps, depicted in Figs. 5(d), 5(e), and 5(f), respectively. As for the intracule distributions, $-\nabla^{2} E^{C}(\mathbf{R})$ maps are discussed in order to allow for a straightforward comparison with $\nabla^{2} E(\mathbf{R})$ and $\nabla^{2} E^{U}(\mathbf{R})$. The $-\nabla^{2} E^{C(\mathrm{HF})}$ map [Fig. 5(d)] reveals that Fermi correlation is important only for interactions involving electrons in the same atom or electrons from neighbor atoms, that is: $\left\{\mathrm{C}_{i i}\right\},\left\{\mathrm{H}_{i i}\right\},\left\{\mathrm{C}_{i j}\right\}$, and $\left\{\mathrm{C}_{i} \mathrm{H}_{i}\right\}$. All these interactions, except for $\left\{\mathrm{H}_{i i}\right\}$, whose contribution overlaps that of $\left\{\mathrm{C}_{i} \mathrm{H}_{i}\right\}$, are characterized as local minima in $-\nabla^{2} E^{C(\mathrm{HF})}$, as well as $\left\{\mathrm{H}_{i} \mathrm{H}_{i}\right\}$. According to the $E^{C}(\mathbf{R})$ values, the minimum associated to $\left\{\mathrm{C}_{i i}\right\}$ is by large the most important, followed by those associated to $\left\{\mathrm{C}_{i} \mathrm{C}_{j}\right\},\left\{\mathrm{C}_{i} \mathrm{H}_{i}\right\}$, and $\left\{\mathrm{H}_{i} \mathrm{H}_{i}\right\}$ (see Table IV). $-\nabla^{2} E^{C(\mathrm{CI})}$ [Fig. 5(e)] is topologically equivalent to $-\nabla^{2} E^{C(\mathrm{HF})}$, except for a new minimum associated to $\left\{\mathrm{H}_{i i}\right\}$. Furthermore, it is found that $\left|E^{C}(\mathbf{R})\right|$ values increase for the $\left\{\mathrm{C}_{i i}\right\}$ intraatomic interaction, but decrease for the interatomic $\left\{\mathrm{C}_{i} \mathrm{C}_{j}\right\}$ and $\left\{\mathrm{C}_{i} \mathrm{H}_{i}\right\}$ interactions, with respect to the HF results.

The $-\nabla^{2} \Delta E^{C(\mathrm{CI}-\mathrm{HF})}$ map [Fig. 5(f)] is consistent with the characteristics of the $-\nabla^{2} E^{C(\mathrm{HF})}$ and $-\nabla^{2} E^{C(\mathrm{CI})}$ distributions discussed above. Thus, upon inclusion of Coulomb correlation, $\left|E^{C}(\mathbf{R})\right|$ increases for intraatomic interactions [negative values in Fig. 5(f)], but decreases for the interatomic $\left\{\mathrm{C}_{i} \mathrm{C}_{j}\right\}$ and $\left\{\mathrm{C}_{i} \mathrm{H}_{i}\right\}$ interactions [positive values in Fig. 5(f)]. This result is consistent with the $-\nabla^{2} \Delta I^{C(\mathrm{CI}-\mathrm{HF})}$ difference map discussed previously, which showed that Coulomb correlation shifts the $I^{C}(\mathbf{r})$ density towards smaller interelectronic distances. Thus, the overall effect of Coulomb correlation is to increase the locality of $\Gamma^{C}$, the correlated component of the electron-pair density. Recently, similar conclusions were reached by means of localization and delocalization indices ${ }^{19}$ and by calculating atomic similarity measures based on the exchange-correlation density. ${ }^{20}$

\section{CONCLUSIONS}

The topologies of the correlated $I^{C}(\mathbf{r})$ and $E^{C}(\mathbf{R})$ and uncorrelated components $I^{U}(\mathbf{r})$ and $E^{U}(\mathbf{R})$ of the intracule and extracule densities have been analyzed and compared to the corresponding total densities $I(\mathbf{r})$ and $E(\mathbf{R})$ for the He, $\mathrm{Ne}$, and $\mathrm{Ar}$ atoms. The same analysis has been carried out for $I(\mathbf{r})$ and its components for the $C_{2}^{-2}, \mathrm{~N}_{2}$, and $O_{2}^{+2}$ molecules. Finally, $\nabla^{2} I(\mathbf{r}), \nabla^{2} E(\mathbf{R})$, and its components have been analyzed for the $\mathrm{C}_{2} \mathrm{H}_{4}$ molecule.

In general, it has been found that $I^{U}(\mathbf{r})$ and $E^{U}(\mathbf{R})$ have the same topological structure as the parent $I(\mathbf{r})$ and $E(\mathbf{R})$ functions. Taking into account that they are derived from a product of one-electron densities, it can be expected that $I^{U}(\mathbf{r})$ and $E^{U}(\mathbf{R})$ will not furnish any additional insight on molecular structure that could not be obtained by means of the one-electron density. On the contrary, $I^{C}(\mathbf{r})$ and $E^{C}(\mathbf{R})$ provide interesting additional information. Local minima in $I^{C}(\mathbf{r})$ and $E^{C}(\mathbf{R})$ (or local maxima in the associated Laplacian functions) correspond only to interactions between electrons which are meaningfully correlated between them. For all systems studied, $I^{C}(\mathbf{r})$ and $\nabla^{2} I^{C}(\mathbf{r})$ distributions reflect that both Fermi and Coulomb electron correlation are short ranged and highly isotropic, while $E^{C}(\mathbf{R})$ and $\nabla^{2} E^{C}(\mathbf{R})$ show that both kinds of correlation decrease considerably the probability of having electron pairs centered around atomic nuclei or bond critical points. Thus, $I^{C}(\mathbf{r})$ and $E^{C}(\mathbf{R})$ distributions or $\nabla^{2} I^{C}(\mathbf{r})$ and $\nabla^{2} E^{C}(\mathbf{R})$ complement each other. This novel analysis of $I^{C}(\mathbf{r})$ and $E^{C}(\mathbf{R})$ yields interesting information regardless of the level of calculation used. Moreover, the comparison of $I^{C}(\mathbf{r})$ and $E^{C}(\mathbf{R})$ distributions (or its Laplacian functions) obtained at the HF and CI levels of theory reveals that exchange or Fermi correlation accounts for the main topological features in these distributions, including the atomic shell structure. In general, the role of increasing the level of calculation (i.e., carrying out CI calculations) is to concentrate more $I^{C}(\mathbf{r})$ or $E^{C}(\mathbf{R})$ density on maxima corresponding to intraatomic interactions, and deplete them on maxima associated to interatomic interactions. The overall effect, with respect to the HF description, is a higher locality of the correlated component of the electronpair density $\Gamma^{C}\left(\mathbf{r}_{1}, \mathbf{r}_{2}\right)$. All in all, properties described in this paper make the analysis of $I^{C}(\mathbf{r})$ and $E^{C}(\mathbf{R})$ densities and their Laplacians a promising tool for the analysis of electron correlation in atoms and molecules. More research in this direction in underway in our laboratory.

\section{ACKNOWLEDGMENTS}

This work has been supported by the Spanish DGES Project No. PB98-0457-C02-01. X.F. benefits from a doctoral fellowship from the University of Girona. 
${ }^{1}$ R. F. W. Bader, Atoms in Molecules: A Quantum Theory (Clarendon, Oxford, 1990).

${ }^{2}$ E. R. Davidson, Reduced Density Matrices in Quantum Chemistry (Academic, New York, 1976).

${ }^{3}$ A. J. Coleman, Int. J. Quantum Chem. 18, 457 (1967).

${ }^{4}$ A. J. Thakkar and V. H. Smith, Jr., Chem. Phys. Lett. 42, 476 (1976).

${ }^{5}$ A. E. Carlsson and N. W. Ashcroft, Phys. Rev. B 25, 3474 (1982); A. J. Thakkar, J. Chem. Phys. 84, 6830 (1986).

${ }^{6} \mathrm{E}$. Valderrama, J. M. Ugalde, and R. J. Boyd, in Advances in the Theory of Many-Electron Densities and Reduced Density Matrices, edited by J. Cioslowski (Kluwer Academic/Plenum, New York, in press).

${ }^{7}$ A. J. Thakkar and N. J. Moore, Int. J. Quantum Chem., Symp. 15, 393 (1981).

${ }^{8}$ A. J. Thakkar, A. N. Tripathi, and V. H. Smith, Int. J. Quantum Chem. 26, 157 (1984).

${ }^{9}$ C. Sarasola, J. M. Ugalde, and R. J. Boyd, J. Phys. B 23, 1095 (1990).

${ }^{10}$ C. A. Coulson and A. H. Neilson, Proc. Phys. Soc. London 78, 831 (1961).

${ }^{11}$ R. J. Boyd and C. A. Coulson, J. Phys. B 7, 1805 (1974).

${ }^{12}$ J. Cioslowski and G. Liu, J. Chem. Phys. 105, 8187 (1996).

${ }^{13}$ X. Fradera, M. Duran, and J. Mestres, J. Chem. Phys. 107, 3576 (1997).

${ }^{14}$ X. Fradera, M. Duran, and J. Mestres, Can. J. Chem. 78, 328 (2000).

${ }^{15}$ J. Cioslowski and G. Liu, J. Chem. Phys. 109, 8225 (1998); J. Cioslowski and G. Liu, ibid. 110, 1882 (1999); J. Cioslowski, G. Liu, J. Rychlewski, W. Cencek, and J. Komasa, ibid. 111, 3401 (1999).

${ }^{16}$ X. Fradera, C. Sarasola, J. M. Ugalde, and R. J. Boyd, Chem. Phys. Lett. 304, 393 (1999).

${ }^{17}$ R. McWeeney, Methods of Molecular Quantum Mechanics (Academic, London, 1969).

${ }^{18}$ R. F. W. Bader, A. Streitwieser, A. Neuhaus, K. E. Laidig, and P. Speers, J. Am. Chem. Soc. 118, 4959 (1996); R. F. W. Bader, S. Johnson, and T.-H. Tang, J. Phys. Chem. 100, 15398 (1996); R. J. Gillespie, D. Bayles, J. Platts, G. L. Heard, and R. F. W. Bader, J. Phys. Chem. A 102, 3407 (1998); R. F. W. Bader, and G. L. Heard, J. Chem. Phys. 111, 8789 (1999).

${ }^{19}$ X. Fradera, M. A. Austen, and R. F. W. Bader, J. Phys. Chem. A 103, 304 (1999).
${ }^{20}$ X. Fradera, M. Duran, and J. Mestres, J. Comput. Chem. (in press).

${ }^{21}$ A. M. Lee and P. M. W. Gill, Chem. Phys. Lett. 313, 271 (1999).

${ }^{22}$ M. W. Schmidt et al., J. Comput. Chem. 14, 1347 (1993).

${ }^{23}$ J. Cioslowski and G. Liu, J. Chem. Phys. 105, 4151 (1996).

${ }^{24}$ The location of local minima in $\nabla^{2} I(\mathbf{r})$ and $\nabla^{2} E(\mathbf{R})$ distributions and its components was carried out by using a gradient minimization procedure. Nuclear geometries were used to generate a suitable starting point for each of the formal electron-electron interactions. An exhaustive search of all the local minima possible in the different intracule and extracule distributions was not attempted.

${ }^{25}$ T. Koga and H. Matsuyama, J. Chem. Phys. 107, 10062 (1997).

${ }^{26}$ T. Koga and H. Matsuyama, J. Phys. B 30, 5631 (1997).

${ }^{27}$ F. Arias de Saavedra, I. Porras, E. Buendía, and F. J. Gálvez, J. Phys. B 28, 3123 (1995).

${ }^{28}$ A. Sarsa, F. J. Gálvez, and E. Buendía, J. Chem. Phys. 109, 7075 (1998).

${ }^{29}$ F. J. Gálvez, E. Buendía, and A. Sarsa, J. Chem. Phys. 111, 3319 (1999).

${ }^{30}$ J. M. Mercero, J. E. Fowler, C. Sarasola, and J. M. Ugalde, Phys. Rev. A 59, 4255 (1999).

${ }^{31}$ However, by using different post-HF wave functions, different conclusions are reached in Refs. 29 and 30 on the effect of electron correlation on atomic counterbalance densities. The same post-HF methodology of Ref. $30(\mathrm{CI})$ is used in the present paper, and similar results are obtained for the counterbalance densities.

${ }^{32}$ R. P. Sagar, A. C. T. Ku, and V. H. Smith, Jr., J. Chem. Phys. 88, 4367 (1988).

${ }^{33}$ Z. Shi and R. J. Boyd, J. Chem. Phys. 88, 4375 (1988).

${ }^{34}$ C. Sarasola, L. Domínguez, M. Aguado, and J. M. Ugalde, J. Chem. Phys. 96, 6778 (1992).

${ }^{35}$ X. Fradera, M. Duran, and J. Mestres, Theor. Chem. Acc. 99, 44 (1998).

${ }^{36}$ J. M. Ugalde and C. Sarasola, Phys. Rev. A 49, 3081 (1994).

${ }^{37}$ M. Solà, J. Mestres, J. M. Oliva, M. Duran, and R. Carbó, Int. J. Quantum Chem. 58, 361 (1996).

${ }^{38}$ At post-HF levels of theory it is generally found that $I(0)$ is smaller than $\langle\rho\rangle / 4$, even though a formal proof of this inequality has not been derived yet. See X. Fradera, M. Duran, E. Valderrama, and J. M. Ugalde, Phys. Rev. A (in press). 